\title{
Scalable Implicit Incompressible Resistive MHD with Stabilized FE and Fully-coupled Newton-Krylov-AMG *
}

\author{
J. N. Shadid ${ }^{1,2}$, R. P. Pawlowski ${ }^{1}$, E. C. Cyr ${ }^{1}$, R. S. Tuminaro ${ }^{1}$, \\ L. Chacon ${ }^{3}$, P. D. Weber ${ }^{1}$
}

\begin{abstract}
The computational solution of the governing balance equations for mass, momentum, heat transfer and magnetic induction for resistive magnetohydrodynamics (MHD) systems can be extremely challenging. These difficulties arise from both the strong nonlinear, nonsymmetric coupling of fluid and electromagnetic phenomena, as well as the significant range of time- and length-scales that the interactions of these physical mechanisms produce. This paper explores the development of a scalable, fully-implicit stabilized unstructured finite element (FE) capability for 3D incompressible resistive MHD. The discussion considers the development of a stabilized FE formulation in context of the variational multiscale (VMS) method, and describes the scalable implicit time integration and direct-to-steady-state solution capability. The nonlinear solver strategy employs Newton-Krylov methods, which are preconditioned using fully-coupled algebraic multilevel preconditioners. These preconditioners are shown to enable a robust, scalable and efficient solution approach for the large-scale sparse linear systems generated by the Newton linearization. Verification results demonstrate the expected order-of-accuracy for the stabilized FE discretization. The approach is tested on a variety of prototype problems, that include MHD duct flows, an unstable hydromagnetic Kelvin-Helmholtz shear layer, and a 3D island coalescence problem used to model magnetic reconnection. Initial results that explore the scaling of the solution methods are also presented on up to $128 \mathrm{~K}$ processors for problems with up to $1.8 \mathrm{~B}$ unknowns on a CrayXK7.
\end{abstract}

\section{Introduction}

The resistive magnetohydrodynamics (MHD) model describes the dynamics of charged fluids in the presence of electromagnetic fields. MHD models describe important phenomena in the natural world

\footnotetext{
* This work was partially supported by DOE NNSA ASC Algorithms effort, the DOE Office of Science AMR program at Sandia National Laboratory under contract DE-AC04-94AL85000, and at Los Alamos National Laboratory under contract DE-AC52-06NA25396.

1 Center for Computer Research, Sandia National Laboratories, Albuquerque, New Mexico, 87185

2 Department of Mathematics and Statistics, University of New Mexico, Albuquerque, NM 87131

3 Los Alamos National Laboratory, Los Alamos, NM 87545
}

Preprint submitted to Comp. Meth. App. Mech. and Eng.; SAND Number: SAND2014-20573 J; 18 January 2016

(C) 2016. This manuscript version is made available under the Elsevier user license

http://www.elsevier.com/open-access/userlicense/1.0/ 
(e.g., stellar interiors, solar flares, and planetary magnetic field generation) and in technological applications (e.g., magnetically confined fusion energy [e.g. ITER - tokamak], and pulsed fusion reactors such as Z-pinch-type devices) [1]. The mathematical basis for the continuum modeling of these systems is the solution of the governing partial differential equations (PDEs) describing conservation of mass, momentum, and energy, augmented by the low-frequency Maxwell's equations. This PDE system is non-self adjoint, strongly coupled, highly nonlinear, and characterized by physical phenomena that span a very large range of length- and time-scales. These interacting, nonlinear multiple time-scale physical mechanisms can balance to produce steady-state behavior, nearly balance to evolve a solution on a dynamical time-scale that is long relative to the component time-scales, or can be dominated by a few fast modes. These characteristics make the scalable, robust, accurate, and efficient computational solution of these systems, over relevant dynamical time-scales of interest, extremely challenging.

In the context of MHD simulations, the dominant computational solution strategies for many years have been the use of explicit [2-5] and partially implicit time integrations methods such as implicitexplicit [6-10], semi-implicit [11-14], and operator-splitting [15,16] techniques. With the exception of fully-explicit strategies, which are limited by numerical stability to follow the fastest normal-mode time-scale, all these temporal integration methods include some implicitness to enhance efficiency by removing one or more sources of numerical stiffness in the problem, either from diffusion or from fastwave phenomena. While these types of techniques currently form the basis for many production-level resistive MHD simulation tools (see e.g. [14,9,17,16,18]), a number of applied mathematics, numerical mathematics, and computational science issues remain. Chief among these are the lack of numerical robustness (due to conditional stability constraints stemming from their partially implicit nature), temporal accuracy (which also limits the usable timestep), and the lack of robust, efficient, scalable nonlinear iterative solution methods (which limits the achievable resolution).

From this point of view, fully-implicit formulations, coupled with effective robust nonlinear iterative solution methods, become attractive, as they have the potential to provide stable, higher-order timeintegration of these complex multiphysics systems when long dynamical time-scales are of interest. These methods can follow the dynamical time-scales of interest, as opposed to time-scales determined by either numerical stability or by temporal order-of-accuracy reduction. However, as described above, the existence of fast normal modes in MHD make the associated algebraic systems very stiff, and thus difficult to solve iteratively using modern iterative techniques. Despite these challenges, much effort has been invested in the last decade towards the development of more fully-implicit, nonlinearly coupled solution methods for MHD, with the goal of enhancing robustness, accuracy, and efficiency (see e.g. $[15,7,6,19-27])$.

In addition to the challenges associated with robustly and efficiently dealing with the myriad of timescales produced by the resistive MHD system, there are a number of spatial discretization issues that add considerable complexity to the numerical approximation of these systems. These include dealing with the dual saddle-point structure of the velocity-pressure $(\mathbf{u}, P)$ interactions in the Navier-Stokes operators, and the satisfaction/enforcement of the solenoidal involution/constraint on the magnetic induction $(\nabla \cdot \mathbf{B}=0)$ in conjunction with the evolution of the induction equation for $\mathbf{B}$. Briefly, in the context of finite volume (FV) and finite element (FE) methods, approaches to deal with these difficulties include: physics-compatible discretizations that enforce directly key mathematical properties of the continuous problem (see e.g. [28-32,22,33]); methods that transform to potential-based formulations to eliminate one or both saddle-point sub-systems $[20,34,9,35,25]$, exact and weighted-exact penalty formulations [36-39], and stabilization methods that consider the Lagrange multiplier form of the solenoidal involution/constraint for the induction equation, and then regularize the double-saddle point structure [40-42].

This study pursues fully-implicit formulations in the context of a specific FE discretization based on 
regularization/stabilization methods. These methods introduce a regularization of the magnetic field saddle-point sub-problem to enforce the solenoidal constraint on the magnetic field $\mathbf{B}$, and generate a system with four unknowns, the three components of $\mathbf{B}$ and the Lagrange multiplier, $r$ [40-42]. This system, combined with the Navier-Stokes sub-system, produces a double-saddle point structure for the resulting MHD system and thus requires compatible spaces for both $(\mathbf{u}, P)$ and $\mathbf{B}$ and $r$ that respect the Ladyzhenskaya-Babuska-Brezzi (LLB) condition. The condition for $(\mathbf{B}, r)$ is analogous to the wellknown constraint on the velocity and pressure spaces $(\mathbf{v}, p)$ in the Stokes flow system (see e.g. [43,44]). In this context, references [41,42] have developed a stabilized FE formulation for resistive MHD in the curl form of the equations with constant properties, and developed weak forms that regularize the double saddle-point structure, and generate streamline upwind Petrov-Galerkin (SUPG) like terms that control oscillation due to convection effects. These authors present a coercivity result for the system that enables development of stabilization parameters that obtain optimal convergence characteristics. It should be noted that an important limitation of these approaches is that they can be shown to be convergent only when the magnetics sub-problem is properly posed in $H^{1}(\Omega)$, which applies when the domain $\Omega$ is convex or its boundary is sufficiently smooth (see e.g. [37,45]). Recently, in [45], a stabilized FE method has been proposed that is unconditionally stable and convergent. This stabilized formulation differs from the approaches above in that it defines separate residuals, stabilization terms, and stabilization parameters for the induction and the Lagrange multiplier and requires use of meshes with particular macro-element structures. With these characteristics, convergence is demonstrated on non-convex domains and to solutions with singularities.

The current study complements previous work by developing a robust, efficient, fully-coupled stabilized FE formulation for 3D resistive MHD with solution methods that enable both scalable fully-implicit transient and direct-to-steady-state solutions. This work is an extension of [25] that demonstrated for the first time a scalable solution method for a $2 \mathrm{D}$ vector potential formulation for fully-implicit viscoresistive incompressible MHD based on stabilized FE. In that study, the induction equation saddlepoint problem is eliminated by use of the $2 \mathrm{D}$ vector potential [25]. The current study presents a full $3 \mathrm{D}$ formulation that deals with the double saddle-point structure of the incompressible visco-resistive MHD system and demonstrates robust and scalable iterative nonlinear solutions based on fully-coupled algebraic multilevel preconditioned Newton-Krylov methods. It should be noted that the proposed stabilized FE formulation has the additional benefit of enabling the use of equal-order interpolation for all quantities. This simplifies the data structures of the parallel unstructured FE code, and the linear algebra interface for the iterative solution methods that are employed.

The remainder of this paper is organized as follows. Section 2 presents our formulation of the resistive incompressible MHD model. The stabilized FE formulation of the governing 2D resistive MHD equations is developed by a variational multiscale (VMS) method and presented in Section 3. In Section 4, a brief overview of the fully-implicit Newton-Krylov solution method is presented with a discussion of the domain decomposition and multilevel preconditioners. Section 5 presents representative orderof-accuracy verification studies and performance, scaling and simulation results for some illustrative resistive MHD problems. Finally, Section 6 closes with a few conclusions.

\section{Resistive MHD Model Equations}

The system of interest for this study is the 3D resistive iso-thermal MHD equations including dissipative terms for the momentum and magnetic induction equations [1]. This model provides a continuum 
description of charged fluids in the presence of electromagnetic fields. The system of equations is:

$$
\begin{gathered}
\frac{\partial(\rho \mathbf{u})}{\partial t}+\nabla \cdot[\rho \mathbf{u} \otimes \mathbf{u}-\mathcal{T}]=\mathbf{0} \\
\frac{\partial \rho}{\partial t}+\nabla \cdot[\rho \mathbf{u}]=0 \\
\frac{\partial \mathbf{B}}{\partial t}+\nabla \cdot\left[\mathbf{u} \otimes \mathbf{B}-\mathbf{B} \otimes \mathbf{u}-\frac{\eta}{\mu_{0}}\left(\nabla \mathbf{B}-(\nabla \mathbf{B})^{T}\right)\right]=\mathbf{0}
\end{gathered}
$$

Here $\mathbf{u}$ is the plasma velocity; $\rho$ is the ion mass density; $\mathcal{T}$ is the stress tensor; $\mathbf{B}$ is the magnetic induction (here after also termed the magnetic field) that is subject to the divergence-free involution $\nabla \cdot \mathbf{B}=0$. The associated current, $\mathbf{J}$, is obtained from Ampère's law as $\mathbf{J}=\frac{1}{\mu_{0}} \nabla \times \mathbf{B}$. In its simplest form, the resistive MHD equations are completed with definition of the total stress tensor, $\mathcal{T}=\mathbf{T}+\mathbf{T}_{M}$ composed of the fluid and magnetic stress tensors, $\mathbf{T}, \mathbf{T}_{M}$ that are given by,

$$
\begin{gathered}
\mathbf{T}=-P \mathbf{I}+\boldsymbol{\Pi}=-P \mathbf{I}+\mu\left[\nabla \mathbf{u}+\nabla \mathbf{u}^{T}\right], \\
\mathbf{T}_{\mathbf{M}}=\frac{1}{\mu_{0}} \mathbf{B} \otimes \mathbf{B}-\frac{1}{2 \mu_{0}}\|\mathbf{B}\|^{2} \mathbf{I} .
\end{gathered}
$$

Here, $P$ is the plasma pressure, $\boldsymbol{\Pi}$ is the viscous stress tensor, and $\mathbf{I}$ is the identity tensor. The physical parameters in this model are the plasma viscosity, $\mu$, the resistivity, $\eta$, and the magnetic permeability of free space, $\mu_{0}$. Finally, for convenience of notation, a tensor induction flux, $\mathcal{F}$, that is defined as

$$
\mathcal{F}=\mathbf{u} \otimes \mathbf{B}-\mathbf{B} \otimes \mathbf{u}-\frac{\eta}{\mu_{0}}\left(\nabla \mathbf{B}-(\nabla \mathbf{B})^{T}\right)+\psi \mathbf{I}
$$

is introduced.

Satisfying the solenoidal involution $\nabla \cdot \mathbf{B}=0$ in the discrete representation to machine precision is a topic of considerable interest in both structured and unstructured finite-volume and unstructured finite-element contexts (see e.g. $[5,46,22]$ ). In the formulation discussed in this study, the approach of introducing a scalar Lagrange multiplier into the induction equation that allows enforcement of the solenodial involution as a divergence free constraint on the magnetic field is employed. This procedure is common in both the finite volume (see e.g. $[46,5,22]$ ) and in finite element methods (see e.g. [41,42]). This study focuses on the incompressible limit of this system with $(\nabla \cdot \mathbf{u}=0)$. This limit is useful in the modeling of various applications such as low-Lundquist-number liquid-metal MHD flows [47,48], and high-Lundquist-number, large-guide-field fusion plasmas [49-51]. This limit is characteristic of low flow-Mach-number applications for compressible systems as well, and is the most challenging algorithmically because of the presence of the elliptic incompressibility constraint. However, it should be noted that the stabilized FE formulation that is presented, as well as the strongly-coupled Newton-Krylov nonlinear iterative solvers, and the fully-coupled algebraic multilevel preconditioners also work in the variable density low-Mach-number compressible case. Table 1 presents the 3D resistive MHD equations in residual form with the introduction of the scalar Lagrange multiplier and the incompressibility assumption.

\section{A Stabilized Finite Element Formulation for 3D Resistive MHD}

The continuous PDE problem, defined by the 3D resistive MHD equations in Table 1, is approximated by a stabilized FE formulation. This formulation allows for stable equal-order velocity-pressure interpolation and provides for convection stabilization, as described below. 


\begin{tabular}{|l|c|}
\hline Momentum & $\mathbf{R}_{m}=\rho \frac{\partial \mathbf{u}}{\partial t}+\rho \nabla \cdot[\mathbf{u} \otimes \mathbf{u}-\mathcal{T}]=\mathbf{0}$ \\
\hline $\begin{array}{l}\text { Continuity } \\
\text { Constraint }\end{array}$ & $R_{P}=\rho \nabla \cdot \mathbf{u}=0$ \\
\hline $\begin{array}{l}\text { Magnetic } \\
\text { Induction }\end{array}$ & $\mathbf{R}_{I}=\frac{\partial \mathbf{B}}{\partial t}+\nabla \cdot \mathcal{F}=\mathbf{0}$ \\
\hline $\begin{array}{l}\text { Solenodial } \\
\text { Constraint }\end{array}$ & $R_{\psi}=\nabla \cdot \mathbf{B}=0$ \\
\hline
\end{tabular}

Table 1

Residual form of governing incompressible resistive 3D MHD equations. The primitive variables are the velocity vector $\mathbf{u}$, the hydrodynamic pressure $P$, the magnetic induction $\mathbf{B}$, and the Lagrange multiplier $\psi$.

We employ stabilized FE methods to avoid stability and algorithmic limitations of mixed Galerkin FE formulations. In particular, in a mixed Galerkin FE formulation of the momentum-continuity equations of the Navier-Stokes part of the MHD system, there is a stability requirement that the discrete spaces satisfy the the Ladyzhenskaya-Babuska-Brezzi (LBB) stability condition (see e.g. [43] or [44]).This condition prevents the use of equal-order finite element spaces, defined with respect to the same partition of the computational domain in finite element. This same stability requirement is also present in the linkage of the induction-solenoidal-constraint equations for the magnetic field $\mathbf{B}$ and the scalar $\psi[41,42]$. Finally, an additional difficulty is that the mixed Galerkin formulation is prone to instabilities for highly convected flows, even if the LBB condition is satisfied by the finite element spaces.

Consistently stabilized ${ }^{4}$ finite element methods for Navier-Stokes address the issues above by using a combination of properly weighted residuals of the governing balance equations. These methods simultaneously relax the incompressibility constraint and add streamline-diffusion (and sometimes nonlinear discontinuity-capturing-type operators) to the weak equations to limit oscillations in highly convected flows $[52,53]$.

\subsection{Brief outline of 3D Resistive MHD Variational Multiscale Formulation}

This subsecstion briefly considers the governing incompressible resistive 3D MHD equations as defined in Table 1. The discussion that follows outlines the the essential aspects of the development of the stabilized FEM formulation. More details on the general development of variational multiscale (VMS) methods can be found in [54-56]. In this formulation, the weak form solution that is developed will seek solutions in the function space $\mathcal{V}$ defined as

$$
\mathcal{V}=\left\{\mathbf{U} \mid \mathbf{U}=[\mathbf{u}, P, \mathbf{B}, \psi]^{T}, \mathbf{u}, \mathbf{B} \in H^{1}(\Omega), P, \psi \in L_{2}(\Omega)\right\} .
$$

The equivalent Galerkin variational form is then defined as: Find $\mathbf{U} \in \mathcal{V}$ such that

$$
\mathcal{A}(\mathbf{W}, \mathbf{U})=(\mathbf{W}, \mathbf{F}) \quad \forall \mathbf{W} \in \mathcal{V} .
$$

Here, the combined solution vector is $\mathbf{U}=[\mathbf{u}, P, \mathbf{B}, \psi]^{T}$, and $\mathbf{W}=[\mathbf{w}, q, \mathbf{C}, s]^{T}$ is a vector of weighting functions. The semilinear form $\mathcal{A}(\mathbf{W}, \mathbf{U})$ is given by:

$$
\mathcal{A}(\mathbf{W}, \mathbf{U})=\int_{\Omega} \rho \mathbf{w} \cdot \frac{\partial \mathbf{u}}{\partial t} d \Omega-\int_{\Omega} \rho \mathbf{u} \otimes \mathbf{u}: \nabla \mathbf{w} d \Omega+\int_{\Omega} \mathcal{T}: \nabla \mathbf{w} d \Omega+\int_{\Gamma} \mathcal{T} \hat{\mathbf{n}} \cdot \mathbf{w} d \Gamma-\int_{\Gamma} \rho(\mathbf{u} \cdot \hat{\mathbf{n}}) \mathbf{u} \cdot \mathbf{w} d \Gamma
$$

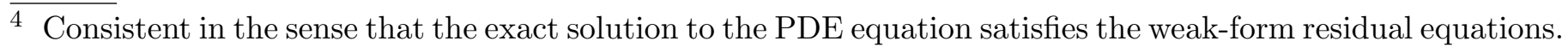




$$
+\int_{\Omega} \rho q \nabla \cdot \mathbf{u} d \Omega+\int_{\Omega} \mathbf{C} \cdot \frac{\partial \mathbf{B}}{\partial t} d \Omega-\int_{\Omega} \mathcal{F}: \nabla \mathbf{C} d \Omega+\int_{\Gamma} \mathcal{F} \hat{\mathbf{n}} \cdot \mathbf{C} d \Gamma+\int_{\Omega} s \nabla \cdot \mathbf{B} d \Omega .
$$

It should be noted that in the above weak form the magnetics surface term $\int_{\Gamma} \mathcal{F} \hat{\mathbf{n}} \cdot \mathbf{C} d \Gamma$ can be shown to be equivalent to $-\int_{\Gamma}(\mathbf{E} \times \hat{\mathbf{n}}) \cdot \mathbf{C} d \Gamma$ when the boundary condition $\psi=0$ on $\Gamma$ has been used.

It is well known that the Galerkin weak form suffers from a number of deficiencies, as described in Section 3 for incompressible resistive MHD. These include the inability to employ equal-order interpolation for velocity/pressure $(\mathbf{u}, P)$, and the magnetic inductance / Lagrange multiplier $(\mathbf{B}, \psi)$ coupling $[25,41]$, as well as the control of unphysical oscillations due to convection and unresolved internal and boundary layers $[41,25,42,57]$. To develop a stabilized finite-element variational weak form that overcomes these deficiencies, a VMS formulation is developed following the general procedures developed and outlined in [54-56]. Following the VMS approach, in the context of FE methods, an additive decomposition of the solution and the weighting function space is introduced

$$
\begin{aligned}
\mathbf{U} & =\mathbf{U}^{h}+\mathbf{U}^{\prime}, \\
\mathbf{W} & =\mathbf{W}^{h}+\mathbf{W}^{\prime}
\end{aligned}
$$

where the exact solution is decomposed into a resolved or coarse scale representation defined by the finite element solution, $\mathbf{U}^{h}$, and the unresolved or sub-grid scale, $\mathbf{U}^{\prime}$ with a similar decomposition of the weighting function $\mathbf{W}$. Corresponding to this decomposition is a splitting of the function space $\mathcal{V}=\mathcal{V}^{h}+\mathcal{V}^{\prime}$ into a finite dimensional resolved-scale subspace and the infinite dimensional unresolvedscale subspace. Introducing this decomposition into the weak form Eqn. (8) and using the linearity property of the semilinear form for the weighting function, and the linear independence of $\mathbf{W}^{\mathbf{h}}$ and $\mathbf{W}^{\prime}$, produces two weak form equations for the resolved and sub-grid scale solutions as:

$$
\begin{array}{ll}
\mathcal{A}\left(\mathbf{W}^{h}, \mathbf{U}^{h}+\mathbf{U}^{\prime}\right)=\left(\mathbf{W}^{h}, \mathbf{F}\right) & \forall \mathbf{W}^{h} \in \mathcal{V}^{h} \\
\mathcal{A}\left(\mathbf{W}^{\prime}, \mathbf{U}^{h}+\mathbf{U}^{\prime}\right)=\left(\mathbf{W}^{\prime}, \mathbf{F}\right) & \forall \mathbf{W}^{\prime} \in \mathcal{V}^{\prime} .
\end{array}
$$

Remark 1 In the subsequent definition of the VMS weak form, the following common practice assumptions will be used [54,55,58,56]

- The fine scale problem (13) is localized to element interiors by assuming $\mathbf{U}^{\prime}=\mathbf{0}$ on element boundaries.

- The unresolved scales are not evolved in time (see Codina for alternate approaches [59]).

- A localized approximation of the unresolved-scales, defined by Eqn. 13, is further assumed to be given by a computable approximation of the sub-grid scale unknowns and the corresponding residuals as

$$
\mathbf{U}^{\prime}=-\tau \mathbf{P} \mathcal{R}\left(\mathbf{U}^{h}\right) ; \quad \mathcal{R}\left(\mathbf{U}^{h}\right)=\left[\mathbf{R}_{m}\left(\mathbf{U}^{h}\right), R_{P}\left(\mathbf{U}^{h}\right), \mathbf{R}_{I}\left(\mathbf{U}^{h}\right), R_{\psi}\left(\mathbf{U}^{h}\right)\right]^{T}
$$

where $\tau$ is a diagonal matrix of stabilization parameters, $\tau=\operatorname{diag}\left(\hat{\tau}_{m}, \hat{\tau}_{m}, \hat{\tau}_{m}, \hat{\tau}_{P}, \hat{\tau}_{I}, \hat{\tau}_{I}, \hat{\tau}_{I}, \hat{\tau}_{\psi}\right)$ that is formed as an algebraic approximation of the inverse of the differential operator. Finally the effective projection operator $\mathbf{P}$ is taken as the identity and thus induces an overlapping sum decomposition in the function space $\mathcal{V}$.

- In view of the definition of the unresolved scales, in terms of the residuals of the resolved scales, a few results follow: 1) the unresolved scales are driven by the resolved scale residuals and this implies that the exact continuous solution satisfies the system of PDEs and is therefore consistent in this sense, 2) since piecewise linear FE basis functions are employed, the second derivative terms are zero or need to be approximated by a projection as in [60] (these terms are neglected in our development)

- The full VMS formulation fully-couples the coarse-scale and sub-grid scale solutions and contains nonlinear (higher-order) sub-grid scale interaction terms. In the context of Navier-Stokes (see e.g. 
[56]) this type of a formulation has advantages in the context of turbulence modeling. However, the goal in this development is more limited and comprises the stabilization of convective effects and the saddle-point coupling between $(\mathbf{u}, P)$ and $(\mathbf{B}, \psi)$. Therefore, we neglect the coupling of the term $\sum_{e} \int_{\Omega^{e}} \rho \mathbf{u}^{h} \otimes \mathbf{u}^{\prime}: \nabla \mathbf{w}^{h} d \Omega$ in the momentum equation that would complete the cross-stress terms and the higher-order nonlinear terms (often termed Reynolds-stress terms). Additionally, we neglect the cross-component-coupling contributions of $\mathbf{B}^{\prime}$ in the momentum equation through the Lorentz force, and $\mathbf{u}^{\prime}$ in the induction equation generated by the convective terms. Recently, we have included these terms in a VMS incompressible FE MHD turbulence model that also shows promise [61]. In this paper a comparison is presented of the performance of the base saddle-point stabilization + SUPG formulation, the full VMS model, and a direct-numerical-simulation (DNS) for a turbulent MHD vortex problem. The results indicate that for flows that have unresolved sub-grid dynamics the VMS model provides a more accurate approximation of the turbulent total energy spectrum.

With these assumptions the VMS weak form becomes, in terms of both resolved and unresolved scales:

$$
\begin{aligned}
\mathcal{A}\left(\mathbf{W}^{h}, \mathbf{U}^{h}+\mathbf{U}^{\prime}\right) & =\mathcal{A}\left(\mathbf{W}^{h}, \mathbf{U}^{h}\right) \\
& -\sum_{e} \int_{\Omega^{e}} \rho \mathbf{u}^{\prime} \otimes \mathbf{u}^{h}: \nabla \mathbf{w}^{h} d \Omega-\sum_{e} \int_{\Omega^{e}} P^{\prime} \nabla \cdot \mathbf{w}^{h} d \Omega-\sum_{e} \int_{\Omega^{e}} \rho \nabla q^{h} \cdot \mathbf{u}^{\prime} d \Omega \\
& +\sum_{e} \int_{\Omega^{e}}\left[\mathbf{B}^{\prime} \otimes \mathbf{u}^{h}-\mathbf{u}^{h} \otimes \mathbf{B}^{\prime}\right]: \nabla \mathbf{C}^{h} d \Omega-\sum_{e} \int_{\Omega^{e}} \psi^{\prime} \nabla \cdot \mathbf{C}^{h} d \Omega-\sum_{e} \int_{\Omega^{e}} \nabla s^{h} \cdot \mathbf{B}^{\prime} d \Omega .
\end{aligned}
$$

Remark 2 Here the reduced VMS weak form includes contributions from:

- the Galerkin form in Eqn. 8 (first line, right hand side),

- a streamline upwind Petrov-Galerkin (SUPG) operator to stabilize convective effects for momentum (second line, first term),

- a cross coupling $(\mathbf{u}, P)$ commonly included at higher Reynolds numbers for Navier-Stokes (second line, second term),

- the pressure-velocity stabilization term that eliminates oscillatory modes from the null space of the incompressible Navier-Stokes operators for the $(\mathbf{u}, P)$ coupling to allow stable equal-order interpolation (second line, third term),

- the SUPG type operators for the divergence form of convection in the induction equation (third line, first term),

- the weak divergence type Laplacian operator cross-coupling term for $(\mathbf{B}, \psi)$ that further enhances the invertibility of the induction equation for steady state systems (third line, second term),

- and the induction-Lagrange multiplier stabilization term that eliminates oscillatory modes from the null space of the induction / solenoidal constraint equations for the $(\mathbf{B}, \psi)$ coupling to allow stable equal-order interpolation (third line, third term).

As described, the approach outlined above is a reasonably standard application of the VMS method to the divergence form of the Lagrange-multiplier form of the incompressible resistive MHD system. Codina [41,42] presents a more detailed derivation of the VMS formulation, for the curl form of the resistive MHD equations, and provides a coercivity result demonstrating stabilization of the double saddle point system and the convective effects. This reference also develops a definition of the stabilization parameters that yields optimal convergence rates in the context of a particular Picard-linearized form of the nonlinear PDE system. Our development is closely related and works with the divergence form of the induction equation, and as the numerical studies demonstrate, provides for the desired stabilization 


\begin{tabular}{|l|r|}
\hline Momentum & $\mathbf{F}_{\mathbf{u}}^{h}=\int_{\Omega} \mathbf{w}^{h} \cdot \mathbf{R}_{m}^{h} d \Omega+\sum_{e} \int_{\Omega_{e}} \rho \hat{\tau}_{m} \mathbf{R}_{m}^{h} \otimes \mathbf{u}^{h}: \nabla \mathbf{w}^{h} d \Omega+\sum_{e} \int_{\Omega_{e}} \hat{\tau}_{P}\left(\nabla \cdot \mathbf{w}^{h}\right) R_{P}^{h} d \Omega$ \\
\hline Total Mass & $F_{P}^{h}=\int_{\Omega} q^{h} R_{P}^{h} d \Omega+\sum_{e} \int_{\Omega_{e}} \rho \hat{\tau}_{m} \nabla q^{h} \cdot \mathbf{R}_{m}^{h} d \Omega$ \\
\hline $\begin{array}{l}\text { Magnetic } \\
\text { Induction }\end{array}$ & $\mathbf{F}_{I}^{h}=\int_{\Omega} \mathbf{C}^{h} \cdot \mathbf{R}_{I}^{h} d \Omega-\sum_{e} \int_{\Omega_{e}} \hat{\tau}_{I}\left(\mathbf{R}_{I}^{h} \otimes \mathbf{u}^{h}-\mathbf{u}^{h} \otimes \mathbf{R}_{I}^{h}\right): \nabla \mathbf{C}^{h} d \Omega+\sum_{e} \int_{\Omega_{e}} \hat{\tau}_{\psi}\left(\nabla \cdot \mathbf{C}^{h}\right) R_{\psi}^{h} d \Omega$ \\
\hline $\begin{array}{l}\text { Solenoidal } \\
\text { Constraint }\end{array}$ & $F_{\psi}^{h}=\int_{\Omega} s^{h} R_{\psi}^{h} d \Omega+\sum_{e} \int_{\Omega_{e}} \hat{\tau}_{I} \nabla s^{h} \cdot \mathbf{R}_{I}^{h} d \Omega$ \\
\hline
\end{tabular}

Table 2

Stabilized finite element formulation of resistive MHD PDEs, where the residual equations $R_{i}$ are presented in Table 1 and the stabilization parameters $\hat{\tau}_{i}$ are defined in the Appendix in Table 6. Here $\left[\mathbf{w}^{h}, q^{h}, \mathbf{C}^{h}, s^{h}\right]$ are the FE weighting functions. The sum $\sum_{e}$ indicates the integrals are taken only over element interiors $\Omega_{e}$ and integration by parts is not performed.

and provides a well-conditioned system of equation for solution by fully-coupled AMG preconditioned Newton-Krylov methods as described in Section 4. The final VMS/stabilized FE formulation is presented in residual form in Table 2, where the Galerkin terms are not expanded and integrated by parts, for the purpose of compact presentation. The stability parameters $\left(\hat{\tau}_{m}, \hat{\tau}_{P}, \hat{\tau}_{I}\right.$ and $\left.\hat{\tau}_{\psi}\right)$ are based on the formulations of Hughes and Mallet [62], Shakib [63], and Hughes [54] for Navier-Stokes with an adaptation for the coupling of the Lorentz force operator to the momentum equation. The definition of the stabilization parameters are provided in the Appendix in Table 6 for momentum, total mass, induction and the Lagrange multiplier.

\subsection{Observations on the Structure of the VMS/Stabilized FE Resistive MHD Formulation}

This brief subsection considers the structure of the VMS/stabilized FE formulation as presented in Table 2 and the correspondence of this form to a few other well know types of approaches for MHD. In this context the discussion considers the VMS magnetics formulation and its connection to the exact penalty method (see e.g. [44]), divergence cleaning methods (see e.g. [46,5]), and projection methods (see e.g. [64]). This demonstration is intended only to formally present the correspondence of the structure of these systems and not present a detailed analysis of the required assumptions to demonstrate a rigorous equivalence.

\subsubsection{Correspondence to Exact Penalty Formulation and Parabolic Divergence Cleaning Methods}

The exact penalty formulation can be obtained from the VMS form by the following modifications.

(1) The stability parameter, $\hat{\tau}_{\psi}$ is taken as $\hat{\tau}_{\psi}=\eta / \mu_{0}$ in the weak divergence Laplacian type operator in the induction equation.

(2) For sake of compact representation, the SUPG type term is dropped, although this is not required since upwinding this form would also be desirable.

(3) In the presentation of the resulting induction equation the stationary curl form is used to correspond to the Gunzburger et. al. development [44], the residual of the solenoidal constraint is explicitly included, and for the first-order weak divergence Laplacian type operator the specification of not integrating by parts is no longer required. 
(4) Finally rather than explicitly using a Lagrange multiplier to weakly enforce the solenoidal constraint, this equation is dropped by setting $\psi=0$.

The resulting weak form is then

$$
\int_{\Omega} \mathbf{C}^{h} \cdot\left[-\nabla \times\left(\mathbf{u}^{h} \times \mathbf{B}^{h}\right)+\nabla \times\left(\frac{\eta}{\mu_{0}} \nabla \times \mathbf{B}^{h}\right)\right] d \Omega+\int_{\Omega} \frac{\eta}{\mu_{0}}\left(\nabla \cdot \mathbf{C}^{h}\right)\left(\nabla \cdot \mathbf{B}^{h}\right)=\mathbf{0}
$$

This form is then seen to be equivalent to the exact penalty method where inclusion of the weak form divergence Laplacian is proven to yield a well-posedness result for a linearized stationary resistive MHD system, with the use of stable mixed interpolation for $(\mathbf{u}, P)$ such as Q2/Q1 and a nodal discretization (e.g. Q2 or Q1) for B, on a convex domain or sufficiently smooth boundary. With the assumptions above and the following additional assumptions

(1) Sufficient continuity of the fields.

(2) The required integration by parts with the required homogeneous natural boundary conditions,

(3) In this case if we take $\hat{\tau}_{\psi}=c_{p}^{2}$ and drop the superscripts

the associated transient strong-form equation then becomes

$$
\frac{\partial \mathbf{B}}{\partial t}-\nabla \times(\mathbf{u} \times \mathbf{B})+\nabla \times\left(\frac{\eta}{\mu_{0}} \nabla \times \mathbf{B}\right)-c_{p}^{2} \nabla(\nabla \cdot \mathbf{B})=\mathbf{0}
$$

This strong form formally corresponds to the parabolic divergence cleaning method described by Dedner et. al. [46] where $c_{p}$ is related to the speed at which divergence errors are diffused to the boundary. In [46] a detailed wave analysis of a generalized Lagrange multiplier formulation (hyperbolic, parabolic and elliptic) is presented that details the the mechanisms that propagate divergence error, in satisfaction of the solenoidal constraint, to the boundaries of the domain and in effect "cleans" the divergence error. As well, this strong form is similar to the Marder method for controlling divergence errors (however in this case it is for solenoidal constraint on B, rather than the incorporation of Gauss' law into Ampere's law $[65])$.

\subsubsection{Correspondence to Projection and Elliptic Divergence Cleaning Methods}

The projection method of Brackbill and Barnes [64] can also be obtained from the VMS weak form by considering a specific set of assumptions that relax the consistent stabilizing residual form of the weak solenoidal constraint in Table 2 and apply a first-order operator splitting method to obtain the solution to the transient coupled system. Specifically the assumptions include

(1) The stability parameter is taken as $\hat{\tau}_{\psi}=0$ in the weak divergence Laplacian type operator in the induction equation.

(2) For sake of compact representation, the SUPG type term is dropped, although this is not required since upwinding this form would also be desirable.

(3) Finally the form of the consistent residual based VMS term that stabilizes the enforcement of the solenoidal constraint and the saddle-point coupling of $(\mathbf{B}, \psi)$ is relaxed. That is all of the additional VMS coupling terms, with the exception of the weak Laplacian operator on $\psi$, are taken as zero. The formal correspondence of this inconsistent VMS form with the projection method is then obtained by a semi-discrete representation of the induction - Lagrange multiplier system that uses forward Euler approximations for the time derivative terms and a first-order operator splitting time integration of the systems of equations. Again only the equations associated with the magnetics/Lagrange multiplier system are presented in this discussion, and integration by parts that would bring in the boundary 
data (i.e. the right hand-side) is not displayed to keep the presentation compact. For the first-order operator-split semi-discrete weak form the procedure is as follows.

- Step 1: Solve the induction equation for a provisional field $\mathbf{B}^{h *}$ that does not necessarily satisfy the solenoidal constraint by assuming that $\psi^{h}=0$.

$$
\int_{\Omega} \mathbf{C}^{h} \cdot\left[\frac{\partial \mathbf{B}^{h *}}{\partial t}-\nabla \times\left(\mathbf{u}^{h} \times \mathbf{B}^{h *}\right)+\nabla \times\left(\frac{\eta}{\mu_{0}} \nabla \times \mathbf{B}^{h *}\right)\right] d \Omega=\mathbf{0}
$$

- Step2: Given $\mathbf{B}^{h *}$ solve the solenoidal constraint equation for the Lagrange multiplier, $\psi^{h}$, assuming that $\tau_{I}=\Delta t_{n}$ (i.e. the weak Poisson equation )

$$
\int_{\Omega} \nabla s^{h} \cdot \nabla \psi^{h} d \Omega=-\frac{1}{\Delta t_{n}} \int_{\Omega} s^{h}\left(\nabla \cdot \mathbf{B}^{h *}\right) d \Omega
$$

- Step3: Solve remaining terms in induction equation. This is the projection into the divergence free subspace.

$$
\int_{\Omega} \mathbf{C}^{h} \cdot \frac{\partial \mathbf{B}^{h}}{\partial t} d \Omega=-\int_{\Omega} \mathbf{C}^{h} \cdot \nabla \psi^{h} d \Omega
$$

Assuming the required continuity of the magnetic induction and Lagrange multiplier, the associated strong form for this weak form operator-split system can be shown to correspond to the projection method of Brackbill and Barnes [64]. Finally, it should be pointed out that in the context of the fullyimplicit time integration used in this study, and the inconsistent reduced VMS system described here, a valid interpretation of this reduced formulation is that of an elliptic divergence cleaning method as described in Dedner et. al. [46]. These elliptic methods would exhibit an infinite wave-speed for propagation of divergence errors to the boundaries.

\section{Fully-implicit Fully-coupled Solution by Parallel Newton-Krylov Methods}

\subsection{Fully-implicit Time Integration and Direct to Steady-state Solutions}

For multiple-time-scale multiphysics PDE systems such as resistive MHD, fully-implicit methods are an attractive choice that can often provide unconditionally-stable time integration techniques. These methods can be designed with various types of stability properties that allow robust integration of multiple timescale systems without the requirement to resolve the stiff modes of the system (which are not of interest when they do not control the accuracy of time integration [66]). In this study we employ implicit integrators that include a multi-stage singly-diagonally-implicit Runge-Kutta (SDIRK) method, and a multi-step backward differentiation formula (BDF2) method that are second order [66]. The result of a fully-implicit (or a direct-to-steady-state) solution technique is the development of very large-scale, coupled highly nonlinear system(s) that must be solved. Therefore, these techniques place a heavy burden on both the nonlinear and linear solvers and require robust, scalable, and efficient nonlinear solution methods. In this study, Newton-based iterative nonlinear solvers [67] are employed to solve the challenging nonlinear systems that result in this application. These solvers can exhibit quadratic convergence rates independently of the problem size when sufficiently robust linear solvers are available. For the latter, we employ Krylov iterative techniques. A Newton-Krylov (NK) method [68] is an implementation of Newton's method in which a Krylov iterative solution technique is used to approximately solve the linear systems that are generated at each step of Newton's method. Specifically, to solve the nonlinear system $\mathbf{F}(\mathbf{U})=\mathbf{0}$, we seek a zero of $\mathbf{F}: R^{N} \rightarrow R^{N}$ where $\mathbf{U} \in R^{N}$ is a current 
approximate solution. The Krylov iterative solver is applied to the linearized residual equation

$$
\mathbf{J}_{k} \mathbf{s}_{k+1}=-\mathbf{F}_{k},
$$

where $\mathbf{J}_{k}$ is the Jacobian matrix and $\mathbf{F}_{k}$ is the nonlinear residual, both of which are evaluated at the previous Newton step solution $\mathbf{U}_{k}$. The solution for the Newton direction vector, $\mathbf{s}_{k+1}$, is used to update the previous solution in the sequence. For efficiency an inexact Newton method [69,70] is usually employed, whereby one approximately solves (15) by choosing a forcing term $\eta_{k+1}$, and stopping the Krylov iteration when the inexact Newton condition

$$
\left\|\mathbf{F}_{k}+\mathbf{J}_{k} \mathbf{s}_{k+1}\right\| \leq \eta_{k+1}\left\|\mathbf{F}_{k}\right\|
$$

is satisfied. For efficient implementation, an adaptive convergence criteria can be employed [71-73], and to increase robustness, backtracking of the provisional Newton step can be enforced to ensure a sufficient reduction of the nonlinear residual before the step is accepted $[67,73]$.

In this study, the Jacobian matrix $\mathbf{J}_{k}$ that is used for the Jacobian-vector products in the Krylov solvers, and as the basis for computing the preconditioners described in Section 4.2, is formed by automatic differentiation (AD) techniques. These methods are applied to the programmed functions representing the weak form residuals outlined in Table 2 by employing the SACADO package [74] from the Trilinos framework [75]. The specific form of this discrete system is discussed in the Appendix in Section 8.2, along with the impact of the block structure of the Jacobian and the implications for the design of additional solution methods for this system of equations. Finally, by developing a direct-tosteady-state solution capability based on Newton-Krylov type techniques, additional advanced solution methods such as parameter continuation, bifurcation tracking and linear stability analysis algorithms, can be effectively developed to analyze complex nonlinear solution spaces of interest in the design and operation of physical systems of scientific and technological interest (see e.g. [76]).

\subsection{Domain Decomposition and Fully-coupled Multilevel Preconditioners}

\subsubsection{Additive Schwarz Domain Decomposition}

For the considered class of linear systems described above, convergence is not achieved without preconditioning due to ill-conditioning in the underlying matrix equations [77]. In the current work, we consider Schwarz domain decomposition preconditioners, where the basic idea is to decompose the computational domain $\Omega$ into overlapping subdomains $\Omega_{i}$ and then assign each subdomain to a different processor [78]. One application of the algorithm consists of solving on subdomains and then combining these local solutions to construct a global approximation throughout $\Omega$. The $i^{\text {th }}$ subdomain problem is usually defined by enforcing homogeneous Dirichlet boundary conditions on the subdomain boundary, $\partial \Omega_{i}$. In the minimal-overlap case, the algebraic Schwarz method corresponds to block Jacobi where each block contains all degrees of freedom (DOFs) residing within a given subdomain. Convergence is typically improved by introducing overlap, which can be done recursively. A significant drawback of the one-level Schwarz method is its locality. A single application of the algorithm transfers information between neighboring sub-domains. This implies that many repeated applications are required to combine information across the entire domain. Thus, as the number of subdomains increases, the convergence rate deteriorates for standard elliptic problems due to the lack of global coupling [78]. The convergence rate also deteriorates as the number of unknowns per subdomain increases when ILU $(k)$ is used for a subdomain solver. The results that we present in Section 5.2 will demonstrate such deterioration for the specific problems of interest in our study. To improve algorithmic convergence rates, coarse levels can be introduced to approximate global coupling, for example the introduction of just one more verycoarse level representation (a two-level DD method) can significantly improve performance [79,80]. 
The use of a coarser mesh to accelerate the convergence of a one-level Schwarz preconditioner is similar in principle to the use of a sequence of coarser meshes in multigrid methods [81]. Next we describe our multilevel preconditioner approach that introduces a sequence of coarser level representations. In this context the DD methods described above are still used as the parallel smoother in the multilevel method.

\subsubsection{Graph-based fully-coupled Multilevel Preconditioners}

Multigrid methods (e.g., [82-84]) are efficient algorithms for solving linear systems modeling elliptic partial differential equations. The basic idea is to efficiently damp oscillatory error components using high fidelity models while smooth error components are handled with lower resolution versions. An example multigrid iteration is given in Figure 1 . Here, one is interested in solving $A_{1} u=b$. The $A_{\ell}$

$$
\begin{aligned}
\operatorname{MGV}\left(A_{\ell}, u, b, \ell\right): & \\
\text { if } \ell & \neq \ell_{\text {max }} \\
& u \leftarrow \mathcal{S}_{\ell}^{\text {pre }}\left(A_{\ell}, u, b\right) \\
r & \leftarrow b-A_{\ell} u ; c \leftarrow 0 \\
& \leftarrow \operatorname{MGV}\left(A_{\ell+1}, c, P_{\ell}^{T} r, \ell+1\right) \\
& u \leftarrow u+P_{\ell} c \\
& \leftarrow \leftarrow \mathcal{S}_{\ell}^{\text {post }}\left(A_{\ell}, u, b\right) \\
\text { else } & u \leftarrow A_{\ell}^{-1} b
\end{aligned}
$$

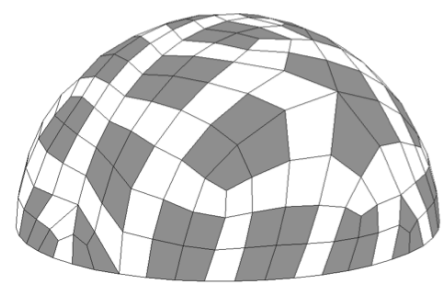

Fig. 1. multigrid V-cycle (left) solving $A_{\ell} u=b$. Aggregation sample (right) where an aggregate includes nodes in and adjacent to one gray region.

$\left(=P_{\ell-1}^{T} A_{\ell-1} P_{\ell-1}\right.$ for $\left.\ell>1\right)$ define a hierarchy of different resolutions. $P_{\ell}$ is an interpolation operator that transfers solutions from level $\ell+1$ to level $\ell$. Finally, $\mathcal{S}_{\ell}^{\text {pre }}$ and $\mathcal{S}_{\ell}^{\text {post }}$ define pre-smoothing and post-smoothing operators, that in most cases correspond to the application of iterative methods that efficiently reduce high-frequency localized error components [82-84]. In this study the same DD $I L U(k)$ algorithm described above is used as a smoother on each level. On the coarsest level the global coupling is represented and a sparse direct solver that handles all of the error-modes is employed (in this case $\operatorname{KLU}[85,86])$.

Construction of the $P_{\ell}$ 's is the most complex aspect. We drop level subscripts and focus on a single interpolation operator within the hierarchy to simplify the discussion. We begin by defining a graph from the $N \times N$ matrix $A$. Graph vertices correspond to matrix rows for scalar PDEs, while for PDE systems one vertex is associated with each nodal block of unknowns, e.g. velocities, pressure, magnetic field and Lagrange multiplier, at a particular grid point. An edge exists between two vertices if there are nonzeros in the block matrix which couples the first vertex's rows with the second vertex's columns, or vice versa. Formally, the graph is $G=(\mathcal{V}, \mathcal{E})$ where $\mathcal{V}=\{1, \ldots, n\}$ and $\mathcal{E}=\left\{(i, j)\right.$ s.t. nnz $\left.\left(A_{i j}\right)+n n z\left(A_{j i}\right)>0\right\}$. Here, $n(=N / m)$ is the number of nodes, $m$ is the number of degrees-of-freedom per node, $n n z(C)$ gives the number of nonzeros in $C$, and the submatrix $A_{i j}$ includes rows of $A$ from $(i-1) m+1$ to $i m$ and columns from $(j-1) m+1$ to $j m .{ }^{5}$ In some situations, it is advantageous to omit edges if all entries within the coupling block are small [87], though this is not considered in this paper.

Constructing $P$ consists of deriving a sparsity pattern and then assigning nonzero values. The pattern

$\overline{5}$ Equations must be ordered so that degrees-of-freedom within a node are consecutive. 
is determined by decomposing graph vertices into aggregates $\mathcal{A}^{i}$ such that

$$
\bigcup_{i=1}^{\hat{n}} \mathcal{A}^{i}=\mathcal{V}, \mathcal{A}^{i} \cap \mathcal{A}^{j}=\emptyset, 1 \leq i<j \leq \hat{n},
$$

where $\hat{n}$ is the number of aggregates and is equal to the number of coarse vertices (as each aggregate gives rise to one coarse vertex). Figure 1 gives an aggregation. Each aggregate normally consists of connected nodes. Typically, aggregates should be approximately the same size and roughly spherical in shape (for isotropic problems). Generally, small aggregates lead to high costs per iteration due to the presence of more levels and generally denser coarse matrices. Larger aggregates lead to poorer multigrid convergence rates. In our work, we target aggregates with a diameter of three. A rudimentary aggregation algorithm is given below.

Basic Aggregation

phase 1: repeat until all unaggregated vertices are adjacent to an aggregate

a) pick root vertex not adjacent to any existing aggregate

b) define new aggregate as root vertex plus all its neighbors

phase 2: sweep unaggregated vertices into existing aggregates or use them

to form new aggregates

An algorithm snapshot might look like Figure 2 where three aggregates have been formed and $\mathcal{A}^{4}$ is being created. After this, only the vertex in the upper left corner can be chosen in phase $1 a$ to create $\mathcal{A}^{5}$. All remaining unaggregated vertices must be addressed in phase 2. Generally, phase 2 must
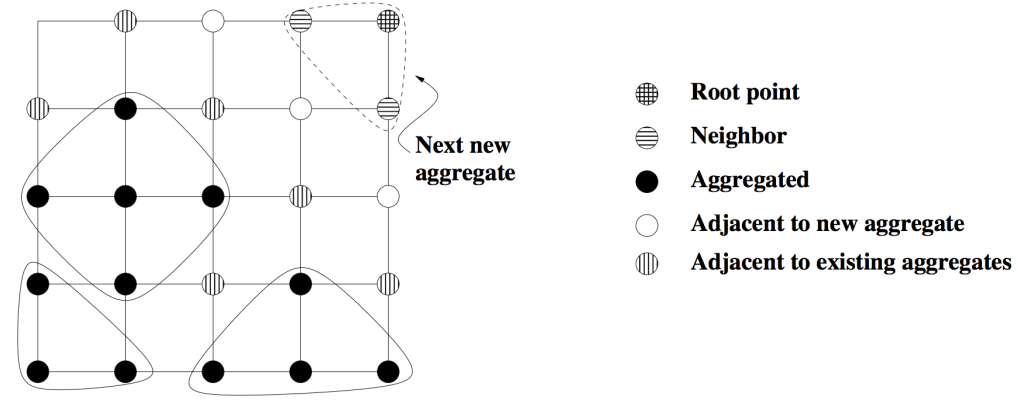

Fig. 2. Snapshot of basic aggregation process.

be implemented with care to create new aggregates when many adjacent unaggregate vertices exist and to equally distribute other remaining vertices among existing aggregates. Overall, the resulting aggregates are less uniform when many vertices are left for phase 2. Thus, a new root should be "close" to existing aggregates (e.g. a distance three from several already chosen root vertices). This efficiently packs aggregates and avoids leaving many holes for phase 2 . Unfortunately, efficient packing is difficult in parallel.

The simplest parallel procedure is where each processor aggregates its local piece of the grid, ignoring connections between processors. Its main disadvantages are that it can produce many small aggregates near inter-processor boundaries and that no aggregate can span two processors, implying that the number of aggregates cannot be less than the number of processors. These disadvantages are pronounced on levels with few vertices per processor and can lead to degradation in the multigrid convergence rate. To limit this, we optionally repartitioning coarse operators. In addition to load balancing, repartitioning enforces some minimum number of vertices per processor (e.g., 1000 vertices) by only employing a processor subset on some coarse levels. The total computational effort associated with these coarse levels is typically small (compared to fine level computations) and dominated by communication. Thus, the convergence improvement and reduced communication costs outweigh any processor inactivity. 
Once the aggregates are chosen a simple grid transfer is defined via

$$
P=\left(\begin{array}{cccc}
I^{1} B & & & \\
& I^{2} B & & \\
& & \ddots & \\
& & & I^{\hat{n}} B
\end{array}\right)
$$

where $B \in \mathbb{R}^{N \times m}$ is a set of $m$ "constant" vectors and the $I^{k}$ are row subsets of the $N \times N$ identity matrix, $I$. Specifically, $I=\left[\begin{array}{lll}I_{1}^{T} & I_{2}^{T} \ldots & I_{\hat{n}}^{T}\end{array}\right]$ where $I_{k} \in \mathbb{R}^{s_{k} \times N}$ and $s_{k}$ is the number of matrix rows within $\mathcal{A}^{k}$, which is $\left|\mathcal{A}^{k}\right| m$ where $\left|\mathcal{A}^{k}\right|$ is the number of vertices in $\mathcal{A}^{k}$. Here, it has been assumed for notational simplicity that vertices have been assigned consecutively to aggregates, i.e. $\mathcal{A}^{1}$ consists of the lowest numbered vertices, and $\mathcal{A}^{2}$ contains the next lowest vertices, etc. $B$ represents modes that must be accurately interpolated. The $j^{\text {th }}$ column contains ones for all rows associated with the $j^{\text {th }}$ degree of freedom within each block corresponding to all grid nodes. Otherwise, the $j^{\text {th }}$ column contains zeros. The actual interpolation is slightly different from (17) in that columns of $P$ are normalized. In particular, a QR factorization is applied to each $I^{k} B$. The $Q$ matrix is used in place of $I^{k} B$ to define interpolation and the $R$ matrix is used to define the coarse "constants" matrix, $B_{2}$, so that $B=P B_{2}$. The multilevel implementation described above is provided by ML $[79,88]$. ML has been employed successfully in a number of applications that include stabilized FE discretizations of Navier-Stokes and transport-reaction type systems [89,76,90], a 2D vector-potential form for resistive MHD [25,91] as well as drift-diffusion systems for semi-conductor modeling [92]. Aztec [93], Ifpack, KLU, ML, KLU, and Zoltan are available through the Trilinos framework [75].

\section{$5 \quad$ Results and Discussion}

In this study, we present two general classes of results. The first in Section 5.1, are for assessment of the accuracy and order-of-accuracy of the stabilized FE formulation described above by a comparison with analytic solutions. The problems in this section include, 1) a steady-state MHD duct flow with strong coupling between the momentum and the Lorentz forces that provides a detailed assessment of the order-of-accuracy of the spatial discretization; and 2) a transient MHD Rayleigh flow and Alfvén wave propagation problem that allows evaluation of the order-of-accuracy of the spatial and temporal discretization of the stabilized FE formulation.

The second class of results, presented in Section 5.2, are intended to present initial representative results for the robustness, efficiency and scalability of the fully-coupled AMG preconditioned NK solution method described above. The problems in this section include, 1) a MHD duct flow that models an idealized 3D MHD generator and is used to study the scalability of the solvers in the context of a direct-to-steady-sate solution method, 2) a classic transient MHD Hydromagnetic Kelvin-Helmholtz stability problem, and 3) a transient magnetic island coalescence problem, of recent scientific interest, for which we present initial results for the scalability of the solution methods.

\subsection{Representative Verification and Order-of-Accuracy Results}

In this subsection, detailed numerical verification results are presented for a set of resistive MHD problems that admit analytic solutions. Two nonlinear convergence criteria are used to ensure that the numerical solution error is below discretization error. The first is a sufficient reduction in the relative nonlinear residual norm, $\left\|F_{k}\right\| /\left\|F_{o}\right\|<10^{-2}$. In general, this requirement is easily satisfied. The second 
convergence criterion is based on a sufficient decrease of a weighted norm of the Newton update vector. The latter criterion requires that the correction, $\Delta \chi_{i}^{k}$, for any variable, $\chi_{i}$, is small compared to its magnitude, $\left|\chi_{i}^{k}\right|$, and is given by

$$
\sqrt{\frac{1}{N_{u}} \sum_{i=1}^{N_{u}}\left[\frac{\left|\Delta \chi_{i}\right|}{\varepsilon_{r}\left|\chi_{i}\right|+\varepsilon_{a}}\right]^{2}}<1,
$$

where $N_{u}$ is the total number of unknowns, $\varepsilon_{r}$ is the relative error tolerance between the variable correction and its magnitude, and $\varepsilon_{a}$ is the absolute error tolerance of the variable correction. Essentially $\varepsilon_{a}$ sets the magnitude of components that are to be considered to be numerically zero. In the verification studies were high accuracies for the solvers are enforced, the relative-error and absolute-error tolerance are $10^{-8}$ and $10^{-10}$ respectively. Each linear system in Newton's method is solved to a level of $\eta=10^{-8}$.

\subsubsection{MHD Duct Flow: Hartmann Verification Problem}

This problem admits an analytical solution [47] which is often used for MHD verification [41,25]. The geometry is taken as a square box with $(x, y) \in[-5,5] \times[-1,1]$. There is an external pressure gradient that drives the flow of $\frac{\partial P}{\partial x}=-G_{0}$, periodic BCs on the inlet and outlet, no-slip velocity and an external magnetic field of $\left(0, B_{0}, 0\right)$ on the top and bottom, and homogeneous Dirichlet boundary conditions on all surfaces for the Lagrange multiplier. The analytic asymptotic solution is of the form $\mathbf{B}=\left(B_{x}, B_{0}, 0\right)$ and $\mathbf{u}=\left(u_{x}, 0,0\right)$, where $B_{y}=B_{0}$ is an applied external magnetic field whose tension retards the flow. The solution is given by

$$
\begin{aligned}
u_{x} & =\frac{G_{0} L^{2}}{\mu H a \tanh [H a]}\left(1-\frac{\cosh [H a y / L]}{\cosh [H a]}\right) \\
B_{x} & =-\frac{G_{0} L^{2} \mu_{0}}{\sqrt{\mu \eta}} \frac{1}{H a}\left(\frac{y}{L}-\frac{1}{\tanh [H a]} \frac{\sinh [H a y / L]}{\cosh [H a]}\right)
\end{aligned}
$$

The order of accuracy of the numerical formulation is computed by comparison with the analytic solution. While the solution is $1 \mathrm{D}$ in character, the numerical solution is computed on a $3 \mathrm{D}$ domain with $\left[128, N_{y}, 1\right]$ elements in the (x,y,z) direction respectively. In Figure 3, profiles from the computed numerical solution are compared with the analytic solution for $u_{x}$ and $B_{x}$ at $x=0$ for various values of $R e=U L / \nu=R e_{m}=\mu_{0} U L / \eta$, where $U$ is the maximum x-direction velocity, and the Hartmann number is $H a=B_{0} L / \sqrt{\rho \nu \eta}$. In this study, we have taken $L=1, \rho=1, \nu=\eta=1, \mu_{0}=1$. The values of $G_{0}$ and $B_{0}$ are then selected to produce the desired Reynolds and Hartmann numbers. Figure 3 shows excellent agreement with the analytical solution. The right image in Figure 3 shows a detailed spatial convergence study that demonstrates the expected second-order accuracy for linear nodal elements.

\subsubsection{MHD Rayleigh Flow and Alfvén Wave Propagation Problem}

This prototype problem also features an analytical solution [47] which has been used for MHD verification [40]. In the classical form, this is a 1D transient problem that has an infinite plate bounding a conducting fluid in a semi-infinite domain above $y=0$. There is an externally applied magnetic field in the $y$-direction with magnitude $B_{0}$. The plate is initially at rest and then is suddenly set in motion with a velocity $v_{x}=U$. A viscous boundary layer flow is developed where the velocity profile is modified from the classical Rayleigh flow profile [94] by the existence of the magnetic field. As the velocity profile is developed, a self-induced magnetic field in the $x$-direction, $B_{x}$, is developed and an Alfvén wave with velocity $A_{0}=B_{0} / \sqrt{\mu_{0} \rho}$ propagates into the fluid. In the numerical solution, the infinite half-space is approximated as a square box with $(x, y) \in[0,5] \times[0,5]$. For the case where the magnetic Prandtl number $\operatorname{Pr}_{m}=\nu / \eta=1$, the analytic asymptotic solution is of the form $\mathbf{B}=\left(B_{x}, B_{0}, 0\right)$ and $\mathbf{v}=\left(v_{x}, 0,0\right)$, and is given by 

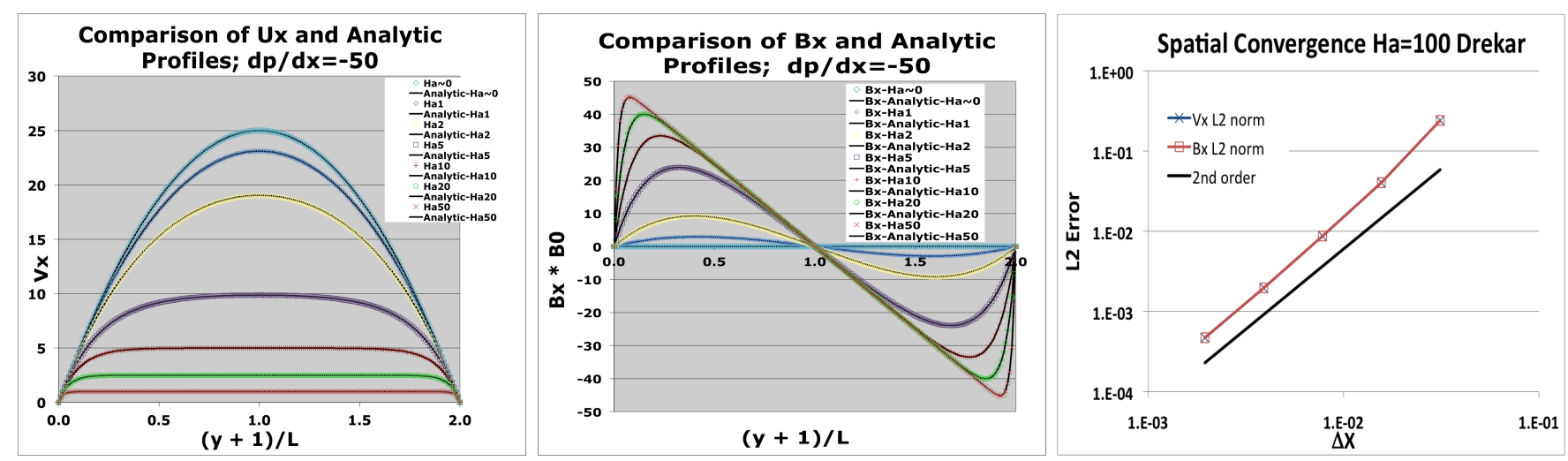

Fig. 3. Hartmann flow verification problem, $R e=R e_{m}$. Figures on the left and center show profiles of analytic solution of $v_{x}$ (left) and $B_{0} B_{x}$ (center) along with numerically computed solutions at $x=0.0$ for a mesh of size $128 \mathrm{x} 128$. The right image is a spatial convergence study.

$$
\begin{aligned}
u_{x}= & \frac{U}{4}\left[e^{\frac{-A_{0} y}{d}}\left(1-\operatorname{erf}\left(\frac{y-A_{0} t}{2 \sqrt{d t}}\right)\right)-\operatorname{erf}\left(\frac{y-A_{0} t}{2 \sqrt{d t}}\right)\right]+ \\
& \frac{1}{4} U\left[e^{\frac{A_{0} y}{d}}\left(1-\operatorname{erf}\left(\frac{y+A_{0} t}{2 \sqrt{d t}}\right)\right)-\operatorname{erf}\left(\frac{y+A_{0} t}{2 \sqrt{d t}}\right)+2\right] \\
B_{x}= & -\frac{1}{4} e^{\frac{-A_{0} y}{d}}\left(-1+e^{\frac{A_{0} y}{d}}\right) U \sqrt{\mu \rho}\left[\operatorname{erfc}\left(\frac{y-A_{0} t}{2 \sqrt{d t}}\right)+e^{\frac{A_{0} y}{d}} \operatorname{erfc}\left(\frac{y+A_{0} t}{2 \sqrt{d t}}\right)\right]
\end{aligned}
$$

The parameters in this problem are taken to be $U=1.0, B_{0}=10, \rho=1, \mu_{0}=1, \eta=1$, and $\mu=1$ with $d=\eta / \mu_{0}=\mu / \rho=1$. The initial conditions are $\mathbf{v}=(0,0,0), P=0, \mathbf{B}=\left(0, B_{0}, 0\right)$. The order of accuracy of the numerical formulation is computed by comparison with the analytic solution. While the solution is $1 \mathrm{D}$ in character, the numerical solution is computed on a $3 \mathrm{D}$ domain with $\left[64, N_{y}, 1\right]$ elements in the $(\mathrm{x}, \mathrm{y}, \mathrm{z})$ direction respectively. In Figure 4, line plots of the analytic and numerical solution for $u_{x}$, and $B_{x}$, are presented at various discrete times. In these plots, the Hartmann layer in $u_{x}$ near the plate surface at $y=0$, and the plateau region influenced by the magnetic field is evident. The figure shows excellent agreement with the analytical solution.

Figure 5 shows a detailed spatial and temporal order-of-accuracy study. A spatial convergence study for $u_{x}, B_{x}$, is shown on the left with a comparison to a second-order reference line for $\Delta t=1.0 \times 10^{-4}$. These results demonstrate the expected second-order spatial order-of-accuracy for linear nodal elements. The image on the right shows convergence of the computed numerical solution for the 2nd order time integrator for $\Delta x=5.0 \times 10^{-3}$. These results verify the second-order temporal order-of-accuracy for the second-order singly diagonal implicit RK method, SDIRK $(2,2)$. As expected for a comparison with analytic results, the $L_{2}$ error norm begins to plateau as the error in the spatial discretization begins to dominate at small time step sizes.

\subsection{Scaling Performance of Krylov Linear Solver and Fully-coupled AMG Preconditioner}

This section briefly describes three prototype resistive MHD problems that are intended to challenge the stabilized FE 3D resistive MHD formulation and the fully-implicit, fully-coupled solution methods presented in this paper. They are also intended to assess the parallel performance (in a weak scaling sense) of these solution methods to very large problem sizes on up to $128 \mathrm{~K}$ cores.

The first problem is a steady-state MHD duct flow configuration representing an idealized MHD gen- 

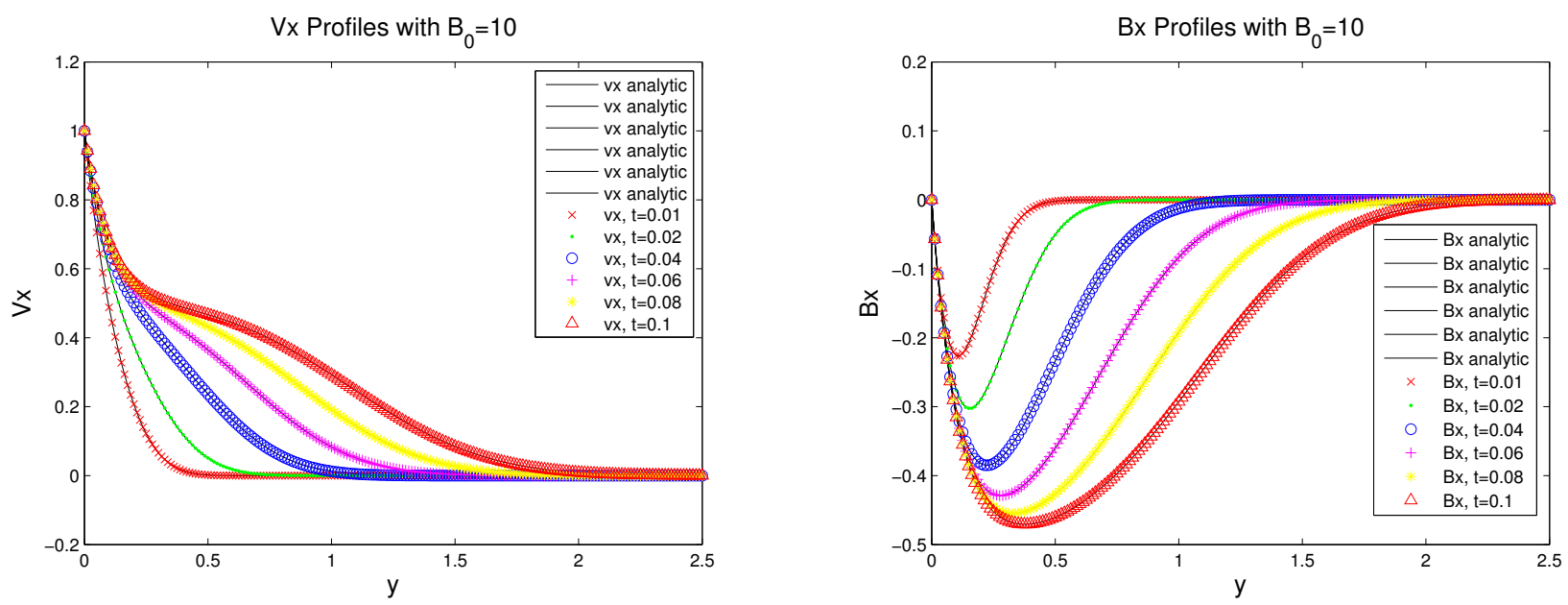

Fig. 4. Modified Rayleigh flow and Alfvén wave verification problem. Figures show plots of $x$-velocity (left) and $x$-magnetic field (right). The mesh has $50 \times 250$ elements and the time step is $5 \times 10^{-4}$ time units.
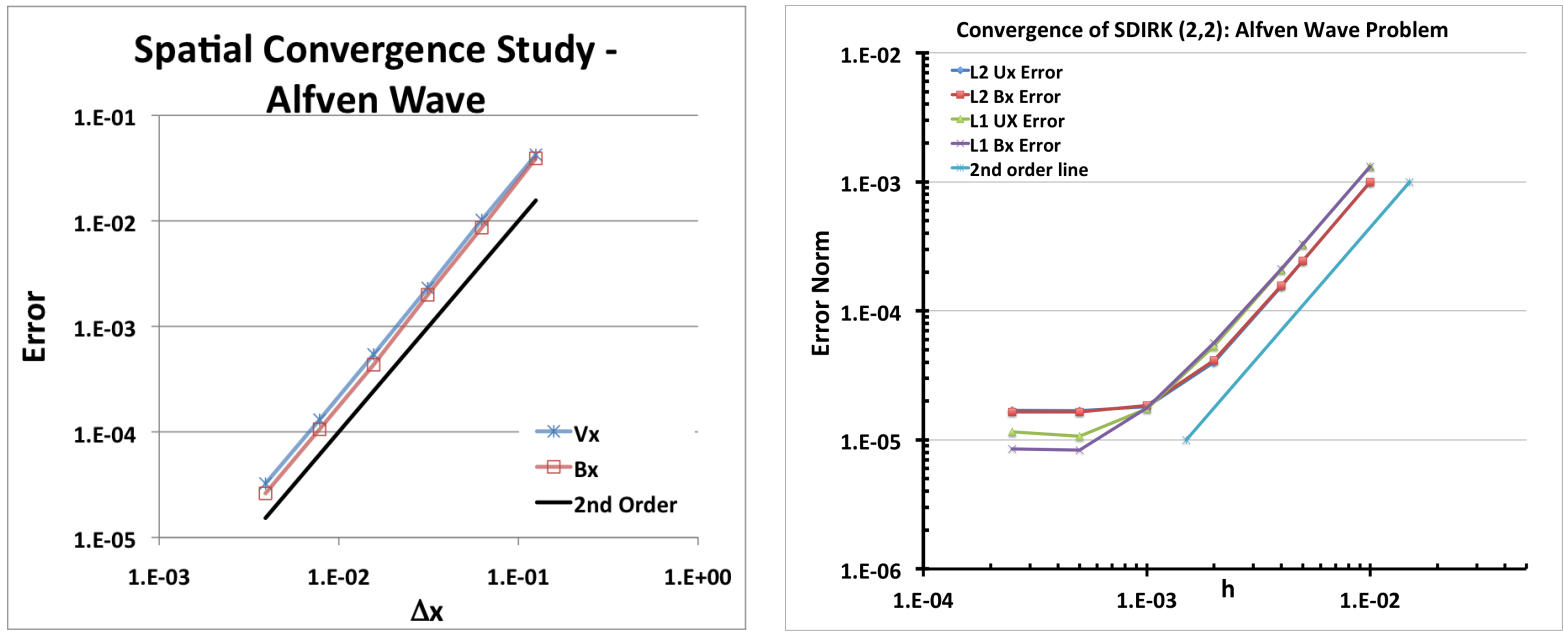

Fig. 5. Order-of-accuracy study for Rayleigh flow and Alfvén wave verification problem.

erator. The second problem is a hydromagnetic Kelvin-Helmholtz-unstable shear-layer problem. The final example is a driven magnetic reconnection problem where a 3D Fadeev magnetic field equilibrium [95], which features islands embedded in a Harris current sheet, undergoes a transient reconfiguration of the magnetic field. The computational timing results for the first two problems presented in this section were obtained on the Titan Cray XK7 at Oak Ridge National Laboratory and were run under the Joule Metric program. The 3D island coalescence magnetic reconnection results were run on the Chama capacity compute server at Sandia National Laboratories. Chama is a capacity cluster, with dual socket Intel Xeon Sandy Bridge processor nodes that are connected by a QDR InfiniBand network. Finally, the Newton convergence criterion is specified in the discussion of each specific problem. In all these examples, the linear systems generated by Newton's method have been left-scaled by a diagonal matrix that contains the inverse of the row sum of absolute values of all entries in the row.

\subsubsection{Steady-state Problem: Idealized MHD Generator}

This problem is a steady-state MHD duct flow configuration representing an idealized MHD generator. The MHD generator induces an electrical current by supplying a set flow-rate of a conducting fluid (mechanical work) through an externally supplied vertical magnetic field. The bending of the magnetic filed lines produces a horizontal electrical current. The geometric domain for this problem is a square 
cross-sectional duct of dimensions $[0,1] \times[0,1] \times[0,15]$. The simple geometry of this problem facilitates scalability studies as different mesh sizes can be easily generated. The velocity boundary conditions are set with Dirichlet inlet velocity of $(U, 0,0)$, no slip on the top, bottom and sides of the channel, and natural boundary conditions on the outflow. The magnetic field boundary conditions on the top and bottom are specified as a set magnetic field configuration with $\left(0, B_{y}^{g e n}, 0\right)$ where

$$
B_{y}^{g e n}=\frac{1}{2} B_{0}\left[\tanh \left(\frac{x-x_{o n}}{\delta}\right)-\tanh \left(\frac{x-x_{o f f}}{\delta}\right)\right]
$$

The inlet, outlet and sides are perfect conductors with $\mathbf{B} \cdot \hat{\mathbf{n}}=\mathbf{0}$ and $\mathbf{E} \times \hat{\mathbf{n}}=\mathbf{0}$, and zero Dirichlet boundary conditions are used on all surfaces for the Lagrange multiplier. This problem has similar characteristics to the Hartmann problem with viscous boundary layers and Hartmann layers occurring at the boundaries, and a flow field that is strongly modified by the magnetic field in the section of the duct where it is active. The parameters in this problem are taken to be $U=1.0, \rho=1, \mu_{0}=1, \eta=1$, $\mu=2.0 \times 10^{-2}, x_{o n}=4.0, x_{o f f}=6.0$, and $\delta=0.1$. The remaining parameters, $B_{0}, \mu, \eta$ are taken to set the Reynolds number, Re, the magnetic Reynolds number of $R e_{m}$ and the Harman number. Figure 6 shows a solution for this problem for $R e=2500, R e_{m}=10$ and $H a=5$. The image shows $u_{x}$ velocity iso-surface colored by $u_{y}$ where the modification of the inlet constant profile and the parabolic profile at the region where the magnetic field is active are evident. Vectors (colored by magnitude) show vertical magnetic field (applied and induced) and horizontal induced current from the bending of the magnetic field lines.

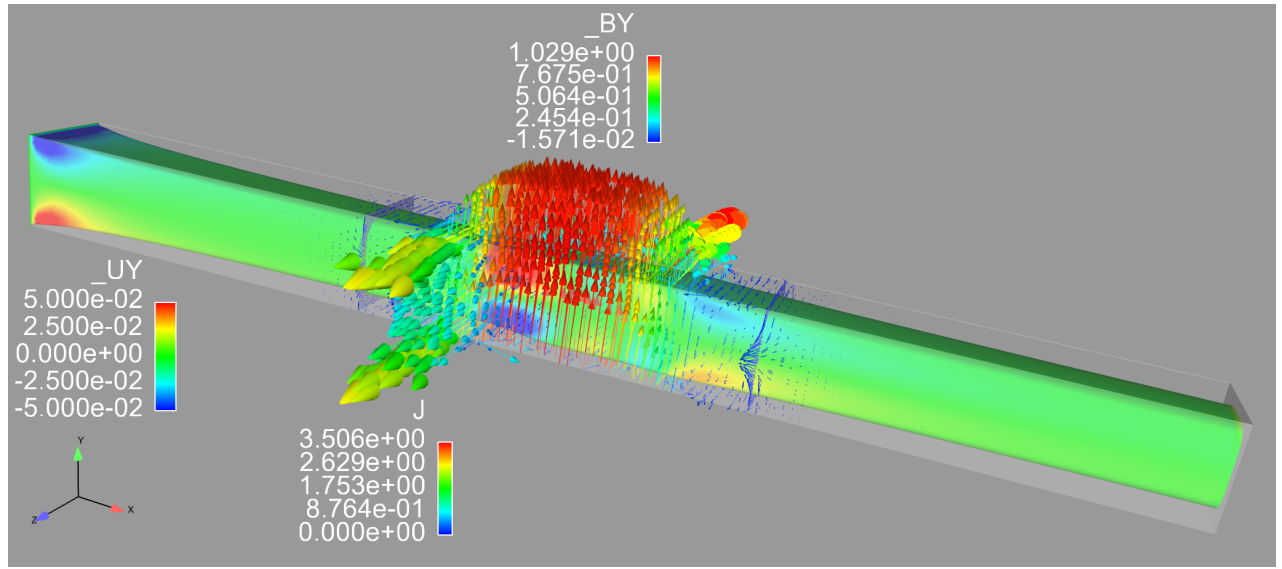

Fig. 6. Idealized 3D MHD Generator Problem. Image shows $u_{x}$ velocity iso-surface colored by $u_{y}$. Vectors (colored by magnitude) show vertical magnetic field (applied and induced) and horizontal induced current.

In this numerical study, the Krylov method is a non-restarted GMRES technique. This choice eliminates the degradation of convergence that can occur from restarting of the GMRES iteration, and allows the scalability of the preconditioners to be directly addressed. For the one-level DD preconditioner, an incomplete factorization ILU $(\mathrm{k})$ sub-domain solver was used with $k=1$ is considered. For the FCAMG preconditioner an ILU(0) smoother is employed and the coarsest problem is solved by the KLU sparse direct solver. For this problem, the inexact Newton forcing term is taken as $\eta_{k+1}=10^{-3}$.

To assess the algorithmic and parallel scaling of the fully-coupled AMG preconditioner we present weak-scaling results for direct-to-steady-state solution of a MHD generator problem with $R e=500$, $R e_{m}=1$ and $H a=2.5$. The results are presented in Table 3 and in Figure 7 for scaling up to $128 \mathrm{~K}$ cores of the Cray XK7. The largest problem size scales to 1.8 billion unknowns and is scaled from 32 to $128 \mathrm{~K}$ cores corresponding to an increase in problem size and core count of 4096x. The results indicate that the fully-coupled AMG preconditioner solution of the 3D stabilized FE resistive MHD formulation 
Table 3

\begin{tabular}{|c|c|c|c|c|c|c|}
\hline \multirow[b]{2}{*}{ proc } & \multirow[b]{2}{*}{ unkns. } & \multicolumn{2}{|c|}{ DD 1-level: ILU(1), ov $=1$} & \multicolumn{3}{|c|}{ FC-AMG: $\operatorname{ILU}(0)$, ov $=1 ; \mathrm{V}(3,3)$} \\
\hline & & $\begin{array}{r}\text { Avg its/ } \\
\text { Newt. } \\
\text { step }\end{array}$ & $\begin{array}{r}\text { Avg. Linear } \\
\text { Solve Time / } \\
\text { Newt. Step }\end{array}$ & $\begin{array}{l}\text { Num. } \\
\text { AMG } \\
\text { levels }\end{array}$ & $\begin{array}{r}\text { Avg its/ } \\
\text { Newt. } \\
\text { step }\end{array}$ & $\begin{array}{r}\text { Avg. Linear } \\
\text { Solve Time / } \\
\text { Newt. Step }\end{array}$ \\
\hline 32 & $497 \mathrm{~K}$ & 73.3 & 6.7 & 4 & 7 & 3.0 \\
\hline 256 & $3.78 \mathrm{M}$ & 165.2 & 18 & 5 & 8.6 & 3.8 \\
\hline 2048 & $29.4 \mathrm{M}$ & 365 & 51.9 & 6 & 12 & 5.6 \\
\hline $16 \mathrm{~K}$ & $232 \mathrm{M}$ & 769 & 156.5 & 7 & 17.7 & 8.9 \\
\hline $128 \mathrm{~K}$ & $1.85 \mathrm{~B}$ & & & 8 & 31.3 & 17 \\
\hline
\end{tabular}

Comparison of 1-level DD and FC-AMG preconditioner for 3D MHD generator problem. The 1 level DD uses an ILU(1) subdomain solver with overlap of 1, the FC-AMG method uses non-smoothed uncoupled aggregation with an ILU(0) smoother with overlap of 1 and with a direct sparse solver on the coarsest problem. The problem takes about 6 Newton steps to solve to steady state.

scales very well with only a slight increase in the average iterations per Newton step for a problem size increase of 4096x. For the $16 \mathrm{~K}$ core, $232 \mathrm{M}$ unknown problem, the FC-AMG solver is about 18x faster than the 1 level DD method.

To consider the robustness of the FC-AMG preconditioner, a set of simulations with increasing Hartmann number were carried out. The results are presented in the lower image of Figure 7 . Over the reported range of Hartman numbers considered, the average linear iteration count per Newton step scales well. While a direct-to-steady-state solution for higher Reynolds numbers $(R e>2500)$ and Hartmann number $(\mathrm{Ha}>64)$ are possible, it is problematic due to the very strong nonlinear coupling. Backtracking strategies, parameter continuation methods, or pseudo-transient time integration would need to be considered [76]. All of these approaches complicate the interpretation of the scaling of the preconditioner and therefore we do not pursue reporting results for these parameter ranges. In the next two examples, the scaling of the preconditioners for transient time integration is considered.

\subsubsection{Transient Problems: Hydromagnetic Kelvin-Helmholtz and Island Coalescence}

Magnetic reconnection is a fundamental process whereby a sheared magnetic field is structurally altered via some dissipation mechanism, resulting in a rapid conversion of magnetic field energy into plasma energy and significant plasma transport. Magnetic reconnection dominates the dynamics of many space and laboratory plasmas, and is at the root of phenomena such as solar flares, coronal mass ejections, plasmoid ejection from the Earth's magnetotail, and major disruptions in magnetic fusion energy (MFE) experiments [96]. The magnetic reconnection problem is intrinsically multiscale in time and space. The key to the computational verification of this regime is the ability to effectively time-integrate the multiple-time-scale resistive MHD system [97]. The next two transient example problems, which are used to evaluate the scaling of the fully-implicit SFE 3D resistive MHD formulation, are MHD system configurations that have been recently used to study magnetic reconnections effects [98,97]. These are

a 3D Hydromagnetic Kelvin-Helmholtz $(\mathrm{KH})$ shear layer and a 3D driven magnetic reconnection island coalescence (IC) problem.

\section{$3 D$ Hydromagnetic Kelvin-Helmholtz Instability}

The specific form of the hydromagnetic Kelvin-Helmholtz unstable shear layer considered here is defined 

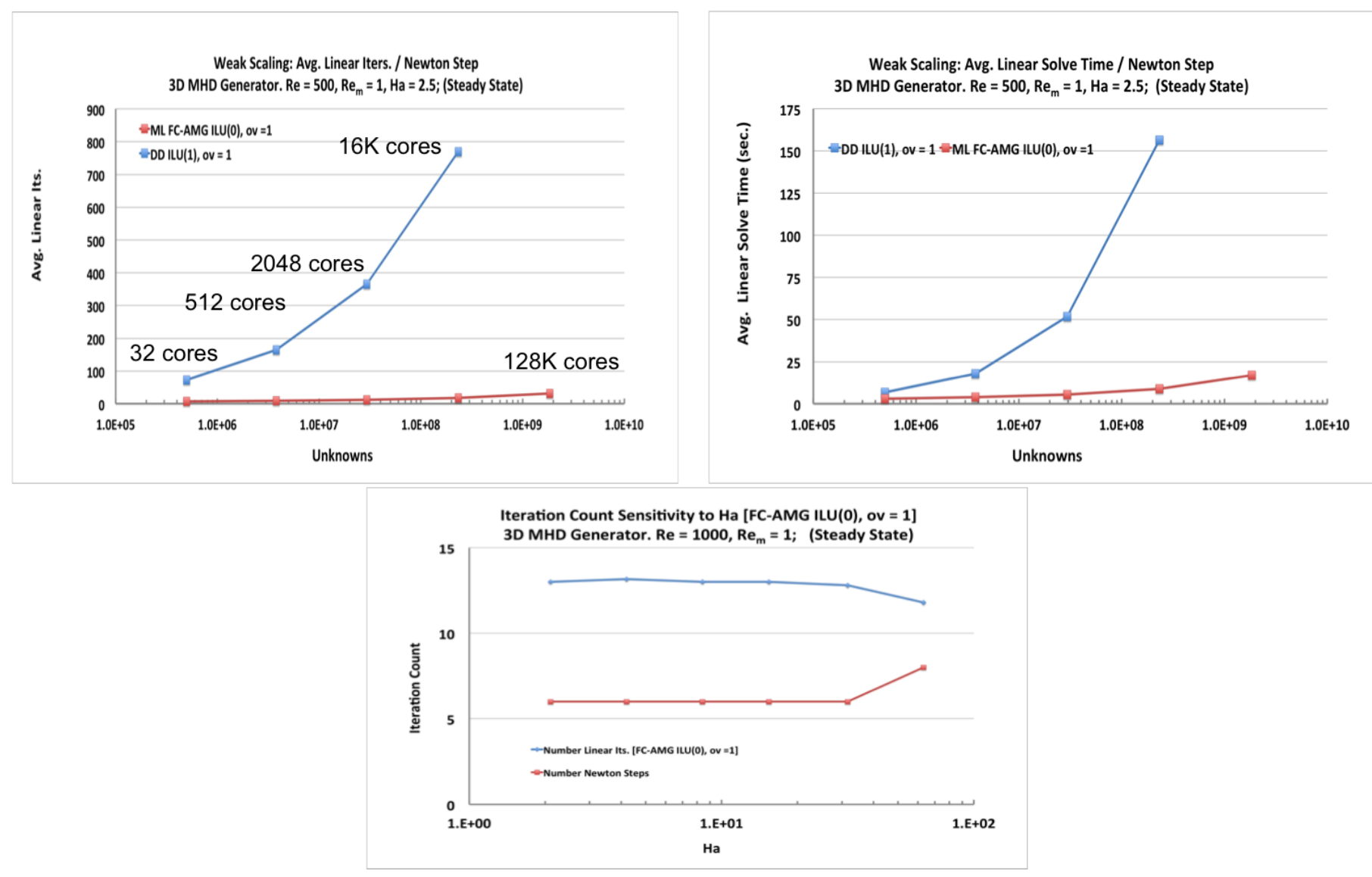

Fig. 7. Idealized 3D MHD Generator Problem. Weak scaling study of average linear iterations per Newton step (upper left), average CPU time per Newton step (upper right), and the scaling with Hartmann number for the average linear iterations per Newton step (lower image).

in a domain of $[0,4] \times[-2,2] \times[0,2]$ and described by an initial condition defined by two counter flowing conducting fluid streams with constant velocities $\mathbf{u}(x, y>0, z, 0)=(1,0,0)$ and $\mathbf{u}(x, y<0, z, 0)=$ $(-1,0,0)$ and a Harris sheet sheared magnetic field defined by

$$
\mathbf{B}(x, y, z, 0)=\left(0, B_{0} \tanh (y / \delta), 0\right) .
$$

The boundary conditions are periodic on the right and left as well as the front and back. The top and bottom are impenetrable for the fluid velocity, the magnetic field is defined by the Harris sheet, and the magnetic Lagrange multiplier is taken as zero. The parameters in this problem are taken to be $\rho=1, \mu_{0}=1, \mu=10^{-4}, \eta=10^{-4}, B_{0}=0.3333, \delta=0.1$ to produce a Reynolds number, $R e=10^{4}$, magnetic Reynolds number, $R e_{m}=10^{4}$ and a super Alfvenic Mach number $M_{A}=u / u_{A}=3$. For these non-dimensional parameters, the shear layer is still Kelvin-Helmholtz unstable, and small instabilities eventually saturate into nonlinear instabilities and form a vortex sheet that evolves with time and undergoes thin current sheet formation, vortex rollup and merging. An image of the unstable flow at time $t=3.9$ is shown in Figure 8 with an iso-vorticity surface colored by the electrical current magnitude and magnetic field vectors. The vorticity surface and the magnetic field vectors indicate the significant reconfiguration of the flow and magnetic field.

In this numerical study, the Krylov method is a non-restarted GMRES technique. For the one-level DD preconditioner, an incomplete factorization ILU $(\mathrm{k})$ sub-domain solver was used, with $k=1$. For the FC-AMG preconditioner, an ILU(0) smoother is employed and the coarsest problem is solved by a sparse direct solver. For this problem, the inexact Newton forcing term is taken as $\eta_{k+1}=10^{-3}$. To 


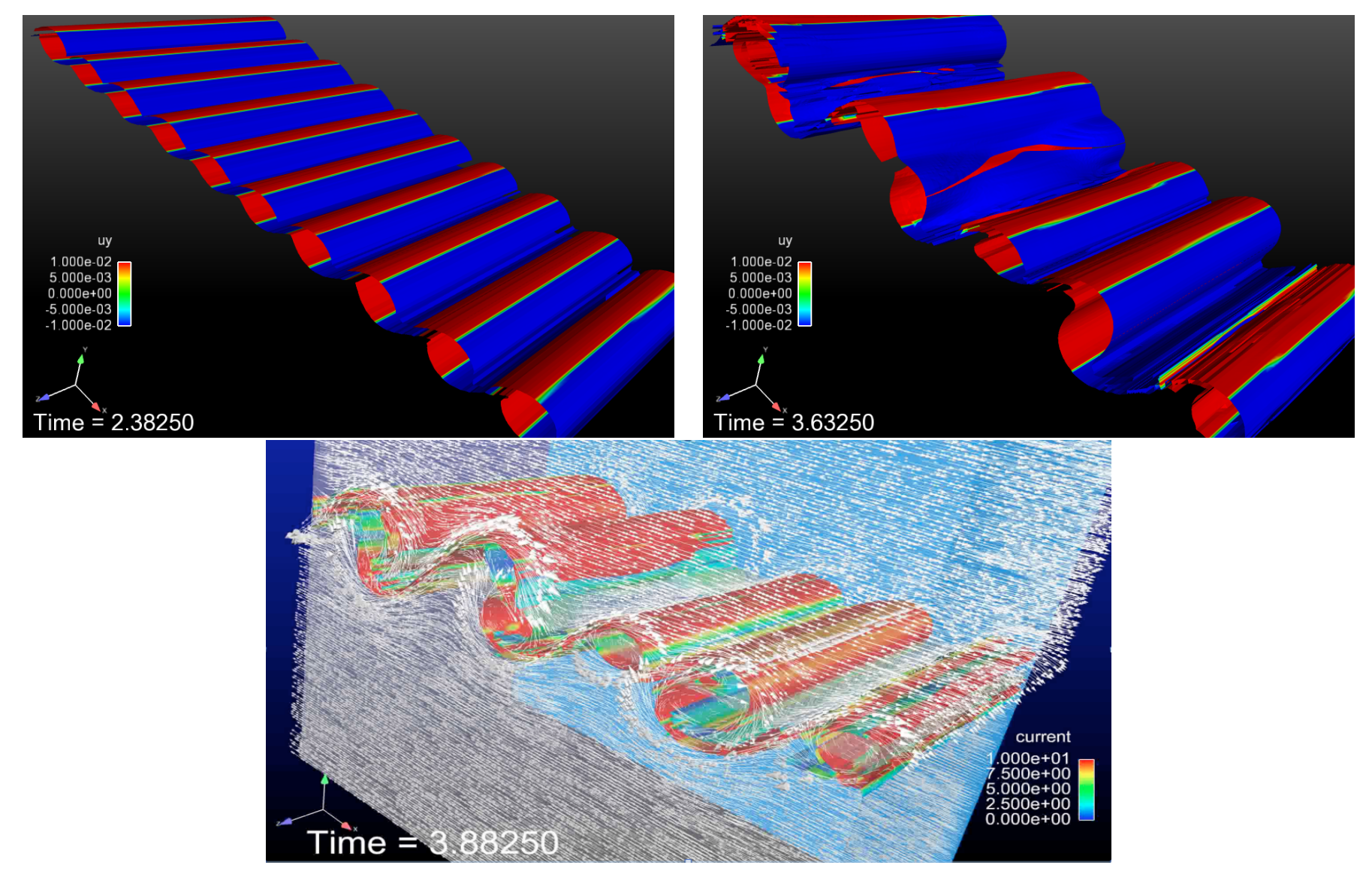

Fig. 8. Hydromagnetic KH problem with $R e=10^{4}, R e_{m}=10^{4}, M_{A}=3$. Iso-vorticity surfaces colored by vertical velocity at time near initial KH instability (upper left), and after vortex-pairs begin to combine (upper right). Iso-vorticity surface colored by plasma current, magnetic field vectors indicate reconnection (lower).

assess the algorithmic and parallel scaling of the fully-coupled, we present weak-scaling results for a transient HMKH problem with $R e=10^{4}, R e_{m}=10^{4}, M_{A}=u / u_{A}=3$ with $C F L=U \Delta t / \Delta x=0.5$ integrated to time $t=2$. The results are averaged over all the time steps, and are presented in Table 4 and in Figure 9 for scaling up to $16 \mathrm{~K}$ cores of the Cray XK7. The largest problem size scales to 164 Million unknowns and is scaled from 32 to $16 \mathrm{~K}$ cores corresponding to an increase in problem size and core count of 512x. The results indicate the the fully-coupled AMG preconditioner solution of the 3D stabilized FE resistive MHD formulation scales very well with only a slight increase in the average iterations per Newton step for a problem increase of 512x. For the $16 \mathrm{~K}$ core, 164M unknown problem the FC-AMG solver is about $5 \mathrm{x}$ faster than the 1 level DD method even for moderate problem size. The $\mathrm{CPU}$ time scaling is also seen to be very good considering the modest problem size per core $(\approx 10 \mathrm{~K}$ unknowns per core).

\section{Magnetic Reconnection in 3D Island Coalescence [24]}

The island coalescence equilibrium is described by the Fadeev solution [95], which features two 3D current tubes (islands) embedded in a Harris current sheet. The structure of this equilibrium can be seen in the upper left plot of Figure 10 with and iso-surface of $\mathbf{J}$. The combined magnetic field produced by the two magnetic islands produces Lorentz forces that pull the islands together, and at finite resistivity the islands coalesce (join) to form one island. Figure 10 shows iso-surfaces in the 3D volume and iso-lines and filled color contours on intersecting planes of $J_{z}$ during the reconnection event. Clearly evident is the formation of the the $\mathrm{x}$-point in the intersecting planes between the islands (see images at $\mathrm{t}=2$ 
Table 4

\begin{tabular}{|c|c|c|c|c|c|c|}
\hline \multirow[b]{2}{*}{ cores } & \multirow[b]{2}{*}{ unkns. } & \multicolumn{2}{|c|}{ DD 1-level: ILU(1), ov $=1$} & \multicolumn{3}{|c|}{ FC-AMG: $\operatorname{ILU}(0)$, ov $=1 ; \mathrm{V}(3,3)$} \\
\hline & & $\begin{array}{r}\text { Avg its/ } \\
\text { Newt. } \\
\text { Step }\end{array}$ & $\begin{array}{r}\text { Avg. Linear } \\
\text { Solve Time / } \\
\text { Newt. Step }\end{array}$ & $\begin{array}{l}\text { Num. } \\
\text { AMG } \\
\text { Levels }\end{array}$ & $\begin{array}{r}\text { Avg its/ } \\
\text { Newt. } \\
\text { Sep }\end{array}$ & $\begin{array}{l}\text { Avg. Linear } \\
\text { Solve Time / } \\
\text { Newt. Step }\end{array}$ \\
\hline 32 & $326 \mathrm{~K}$ & 22.0 & 1.8 & 4 & 2.8 & 1.3 \\
\hline 256 & $2.6 \mathrm{M}$ & 45.0 & 4.0 & 5 & 4.2 & 2.0 \\
\hline 2048 & $20.5 \mathrm{M}$ & 93.0 & 9.2 & 6 & 5 & 2.9 \\
\hline $16 \mathrm{~K}$ & $164 \mathrm{M}$ & 185.0 & 22.0 & 7 & 8.3 & 4.7 \\
\hline
\end{tabular}

Comparison of 1-level DD and FC-AMG preconditioner for 3D Hydromagnetic Kelvin-Helmholtz shear layer problem. The 1 level DD uses an ILU(1) subdomain solver with overlap of 1, the FC-AMG method uses nonsmoothed uncoupled aggregation with an ILU(0) smoother with overlap of 1 and with the KLU direct solver on the coarsest problem. The average number of Newton steps per time steps is about 2 for the simulation.
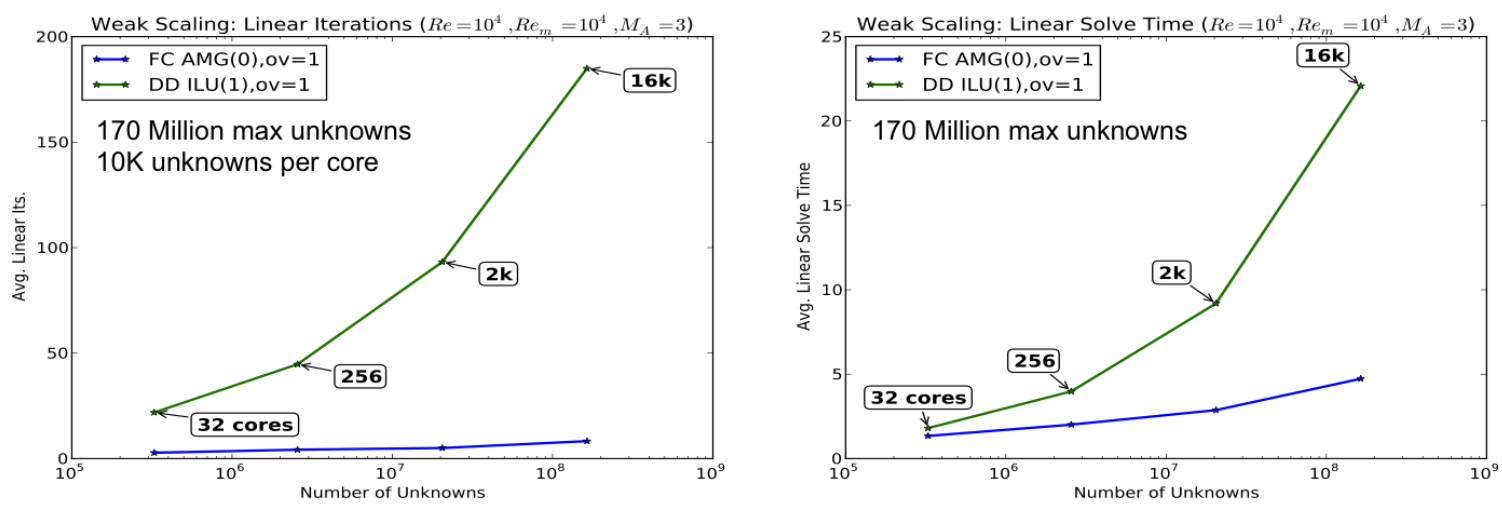

Fig. 9. Hydromagnetic Kelvin-Helmholtz Problem. Weak scaling study of average linear iterations per Newton step (left), average CPU time per Newton step (right).

and 3), the development of thin current sheets at that same x-point location (and the corresponding $3 \mathrm{D}$ surface), and the movement of the center of the tubes (island o-points) towards the x-point [96,97]. The dynamics of island coalescence changes as a function of resistivity. For larger resistivities, the $\mathrm{x}-$ and o-points monotonically approach each other. For low resistivities, fluid-plasma pressure builds up as the islands approach and a sloshing or bouncing of the o-point position is encountered that leads to lower reconnection rates (for more details on the physics see e.g. [96]). Next, a very brief description of the island coalescence setup is presented, with details provided in [24].

This is solved on a domain of $[-1,1] \times[-1,1] \times[-1,1]$. The initial condition for the island coalescence problem consists of zero fluid velocities $\left(\mathbf{u}^{0}=0\right)$, zero Lagrange multipliers $(\psi=0)$ and a Fadeev magnetic equilibrium $[95,24]$ that defines the magnetic field and the fluid pressure. The initial conditions, $\mathbf{B}^{0}=\left(B_{x}^{0}, B_{y}^{0}, 0\right)$, and the resulting balancing plasma fluid pressure, $P^{0}$ are given by

$$
\begin{gathered}
\mathbf{B}^{0}(x, y)=\left(\frac{\sinh \left[\frac{y}{\delta}\right]}{\cosh \left[\frac{y}{\delta}\right]+\epsilon \cos \left[\frac{x}{\delta}\right]}, \frac{\epsilon \sin \left[\frac{x}{\delta}\right]}{\cosh \left[\frac{y}{\delta}\right]+\epsilon \cos \left[\frac{x}{\delta}\right]}, 0\right) \\
P^{0}(x, y)=P_{0}+\frac{\left[1-\epsilon^{2}\right]}{2\left[\cosh \left(\frac{y}{\delta}\right)+\epsilon \cos \left(\frac{x}{\delta}\right)\right]^{2}},
\end{gathered}
$$



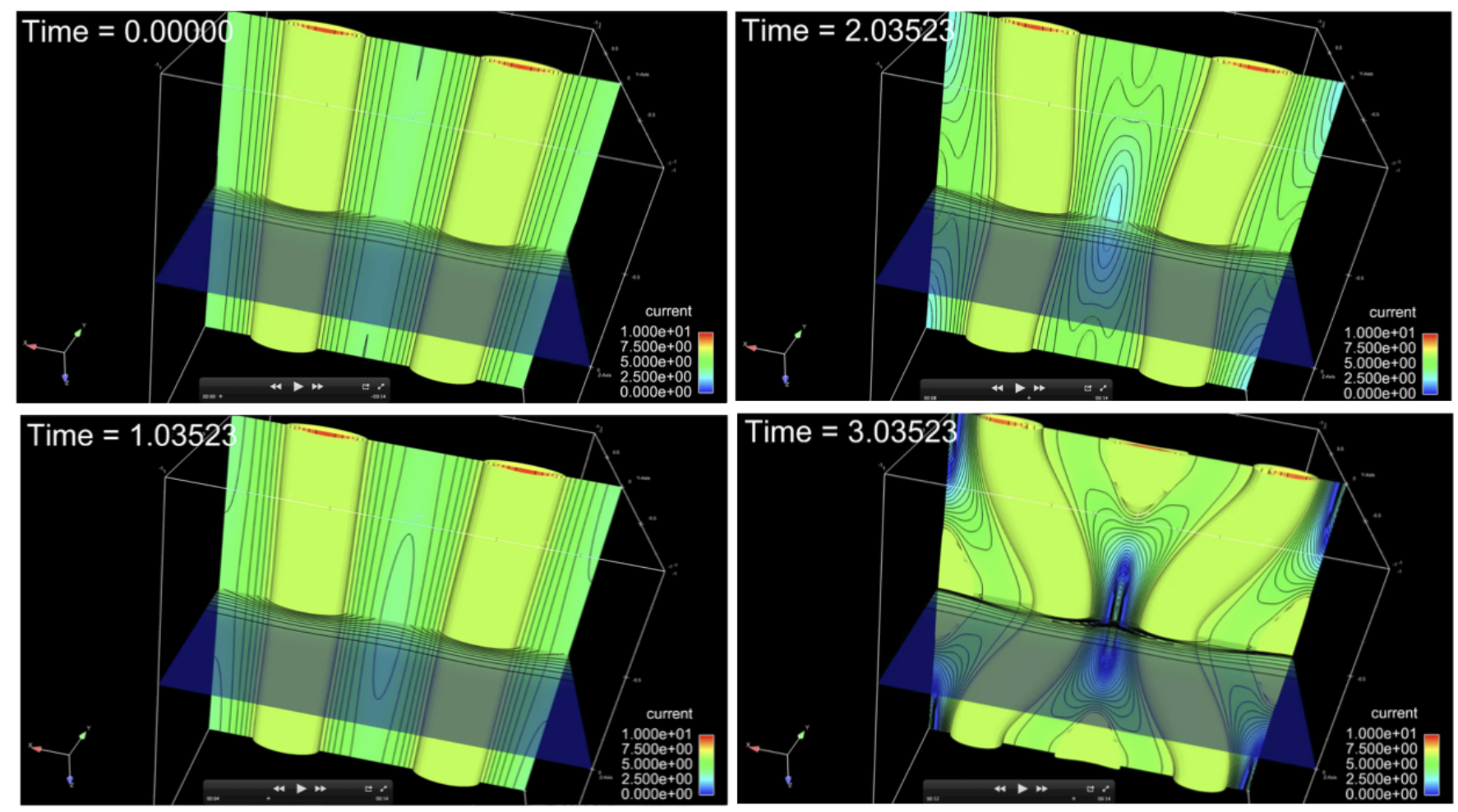

Fig. 10. Structure of the current tubes in 3D island coalescence problem with $S=2 \times 10^{4}$ for the initial condition, and three times in the evolution of the problem $t=1,2,3$. The $3 \mathrm{D}$ current tubes have bent in the z-direction and form current sheets as indicated by the iso-lines and filled color contours of plasma current at five locations.

where $\delta=1 /(2 \pi)$ and $P_{0}=1.0$. To assure that the initial condition is a resistive equilibrium, an external applied electric field in the $z$-direction, $E_{z}^{0}$, of the form

$$
\mathbf{E}^{0}(x, y)=\left(0,0, \frac{\eta\left[1-\epsilon^{2}\right]}{\delta\left[\cosh \left(\frac{y}{\delta}\right)+\epsilon \cos \left(\frac{x}{\delta}\right)\right]^{2}}\right)
$$

is included. The boundary conditions are periodic on the top and bottom as well as the left and right sides. The front and back boundaries are a taken as a perfect conductor $(\mathbf{E} \times \hat{\mathbf{n}}=\mathbf{0}, \mathbf{B} \cdot \hat{\mathbf{n}}=0)$ and the momentum equation surface integral term is used to enforce $P=P_{0}$ and $\mathbf{u} \cdot \hat{\mathbf{n}}=0$. As in all the examples $\psi=0$ is also enfaced on all boundaries. In this study, we have also taken $\rho=1, \nu=\eta=10^{-3}, \mu_{0}=1$ and, using the spacing of the o-points, we have $L=1$. As in [97] these choices imply that the resistivity $\eta=1 / S$, where $S$ is the Lundquist number and is defined as $S=\mu_{0} L V_{A} / \eta$, with $V_{A}=B_{0} / \sqrt{\mu_{0} \rho}$ the Alfven velocity. For the results presented below a 3D perturbation of the magnetic field of the form

$$
\delta \mathbf{B}^{0}(x, y, z)=\Delta\left(\frac{\pi \cos \left(\frac{2 \pi x}{L_{x}}\right) \sin \left(\frac{\pi y}{L_{y}}\right) \cos \left(\frac{2 \pi z}{L_{z}}\right)}{L_{y}}, \frac{2 \pi \sin \left(\frac{2 \pi x}{L_{x}}\right) \cos \left(\frac{\pi y}{L_{y}}\right) \cos \left(\frac{2 \pi z}{L_{z}}\right)}{L_{x}}, 0\right)
$$

is used with $\Delta=10^{-3}$ to set the islands in relative motion in a reproducible manner without relying on long-time roundoff error accumulation.

In this numerical study, the Krylov method is a non-restarted GMRES technique and the FC-AMG preconditioner is a $\mathrm{V}(3,3)$ cycle with $\mathrm{ILU}(0)$ smoother and overlap 1. The KLU sparse direct solver is employed on the coarsest problem. For this problem, the inexact Newton forcing term is taken as $\eta_{k+1}=10^{-3}$. As a preliminary study of the scaling for the FC-AMG preconditioner for the transient solution of the $3 \mathrm{D}$ island coalescence problem at a resistivity of $\eta=10^{-3}$ is presented in Figure 11 . This 
figure presents the weak scaling of the average linear iterations per time step as well as the average of the total time per time step that includes IO. Clearly evident is the excellent algorithmic scaling of the iteration count and total solution time over a problem size increase of 4096. Table 5 details an initial study that considers the robustness of the FC-AMG preconditioned NK solver as a function of Lundquist number. These preliminary results indicate a robust overall solution for a challenging problem over a reasonably wide range of Lundquist numbers.

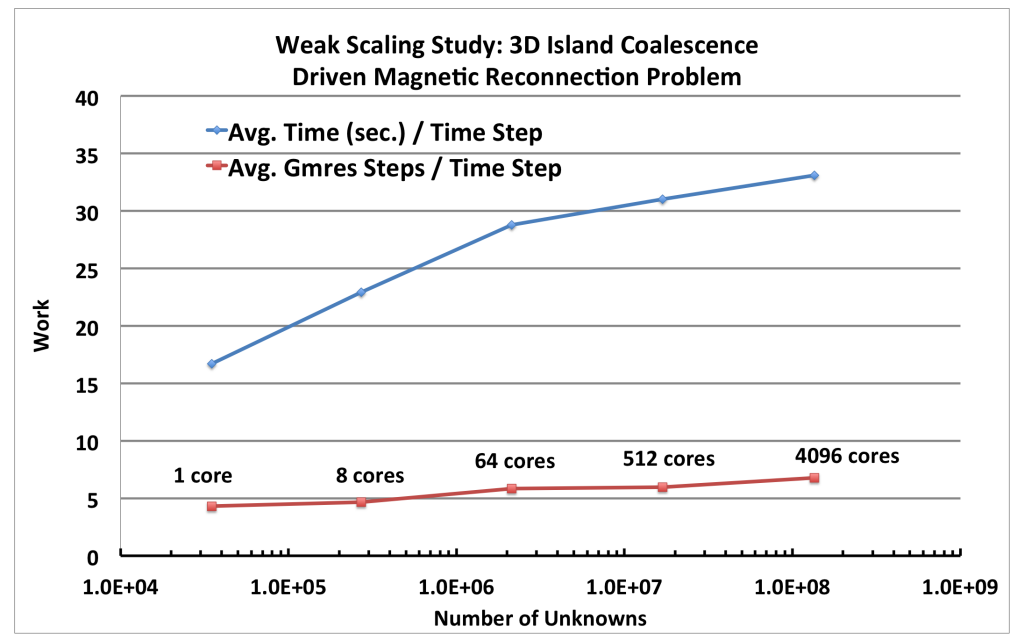

Fig. 11. Driven magnetic reconnection in the Island coalescence problem with a time step of $\Delta t=0.1$ run to a final time of $t=4.0$. Weak scaling study of average linear iterations per time step, and the average total CPU time per time step are shown.

Table 5

\begin{tabular}{|r|c|c|c|c|c|c|c|}
\hline Lundquist No. S & $10^{3}$ & $5 \times 10^{3}$ & $10^{4}$ & $5 \times 10^{4}$ & $10^{5}$ & $5 \times 10^{5}$ & $10^{6}$ \\
\hline \hline Newton Steps/dt & 1.36 & 1.43 & 1.51 & 2 & 2 & 2 & 2 \\
\hline Gmres Steps/dt & 5.2 & 5.7 & 6 & 9.8 & 12 & 8.4 & 8.4 \\
\hline
\end{tabular}

Robustness and scaling of NK FC-AMG preconditioners as a function of Lundquist number. The mesh is $128 \times 128 \times 128(16.9 \mathrm{M}$ unknowns $)$ run on 512 cores. The results are averaged over all time steps to a final time of $t=2.0$ with a time step of $\Delta t=0.0333$.

\section{Conclusions}

This paper has presented a new scalable fully-implicit 3D resistive MHD formulation based on a unstructured stabilized FE formulation. The formulation was briefly presented as developed by a variational multiscale (VMS) development of the divergence form of the governing balance equations. The formulation employed a scalar Lagrange multiplier that was used to enforce the solenodial involution for the magnetic field as a constraint. A critical aspect of the formulation is the stabilization of the two component saddle-point interactions for velocity-pressure $(\mathbf{u}, P)$ and the magnetic field-Lagrange multiplier $(\mathbf{B}, \psi)$ along with the stabilization of convective effects in the momentum and induction equations. The stabilized FE formulation was then verified by order-of-accuracy studies and comparison with analytic solutions for a MHD duct flow (Hartmann problem) and an Alfven wave propagation problem (MHD Rayleigh problem). The solution methods used in this formulation are based on a fully-coupled NewtonKrylov approach employing a fully-coupled algebraic multilevel preconditioner. As an illustration of the 
robustness, scalability, and efficiency of the solution techniques advocated in this paper, performance results have been presented for both low Lundquist number (MHD duct flow), and moderately-high Lundquist number (hydromagnetic Kelvin-Helmholtz and magnetic island coalescence) problems. These results indicate that robust, scalable and efficient solution of challenging multiple-time-scale resistive MHD problems can be obtained with the FC-AMG preconditioned Newton-Krylov solution method.

\section{Acknowledgments}

The authors would like to thank Paul Lin for continued help with improvement of the usability and scaling of the Trilinos suit of solution methods software on large-scale HPC platforms, and David Sondak for suggestions and comments on an early version of the manuscript. The parallel scaling studies were carried out on the DOE/Oak Ridge National Laboratory Jaguar CrayXK7 platform and performed under computing resource allocation from the Joule Metric Effort.

\section{Appendix}

\subsection{Stabilization Parameters}

The specific definition of the stability parameters are provided in Table 6 for momentum, continuity, induction and the solenodial constraint equation. The specific form of the stabilization parameter $\hat{\tau}_{m}$ for the momentum equation is are an adaptation of the quadratic form by Shakib [63] for the Navier-Stokes equations and the addition of a term that balances the Lorentz force in the momentum equation. The form of this term can be determined by a simple method that approximates the velocity developed by the Lorrentz force term in a quasi-static flow. That is, from the steady momentum equation from which the pressure and viscous terms are eliminated, an approximate discrete balance is given by:

$$
\rho V^{2} / h \approx \frac{1}{\mu_{0}} B^{2} / h
$$

the velocity estimate then becomes $V_{A}=B / \sqrt{\rho \mu_{0}}$. This is the Alfven velocity, and this induces an Alfven time scale of $\tau_{A}=V_{A} / h$. Using this estimate the momentum stabilization parameters are given as in Table 6. It should be noted that the Lorentz force term is similar to that used by Codina et. al. for a resistive MHD system [41,42]. Also in the context of the induction equation the standard transientconvection-diffusion form for $\hat{\tau}$ has been modified to include a term that corresponds to approximating the flow velocity by the Alfven velocity $V_{A}$, that is described above. This term has similarity to the Codina et. al. for resistive MHD in [41]. Finally the multidimensional effect of convection is incorporated into the stability parameters by the use of the contravariant metric tensor, $\mathbf{G}_{c}$ (Equation (21)), of the transformation from local element coordinates $\left\{\zeta_{\alpha}\right\}$ to physical coordinates $\left\{x_{i}\right\}$.

$$
\left[\mathbf{G}_{c}\right]_{i j}=\frac{\partial \zeta_{\alpha}}{\partial x_{i}} \frac{\partial \zeta_{\alpha}}{\partial x_{j}}
$$

Shakib [63] considers the one dimensional limiting case of this multidimensional definition for the advection-diffusion equation and presents a comparison with the original SUPG technique. 
Table 6

\begin{tabular}{|l|c|}
\hline Momentum & $\hat{\tau}_{m}=\left[\left(\frac{2 C_{t}^{V} \rho}{\Delta t}\right)^{2}+\rho^{2} C_{c}^{2} \mathbf{u}^{h} \cdot \mathbf{G}_{c} \mathbf{u}^{h}+C_{\mu}^{2} \mu^{2}\left\|\mathbf{G}_{c}\right\|^{2}+C_{B}^{2} \frac{\rho}{\mu_{0}}\left\|\mathbf{B}^{h}\right\|^{2}\left\|\mathbf{G}_{c}\right\|\right]^{-\frac{1}{2}}$ \\
\hline Continuity & $\hat{\tau}_{P}=1 /\left(C_{t}^{V} \operatorname{tr}\left(\mathbf{G}_{\mathbf{c}}\right) \hat{\tau}_{m}\right)$ \\
\hline $\begin{array}{l}\text { Magnetic } \\
\text { Induction }\end{array}$ & $\hat{\tau}_{B}=\left[\left(\frac{2 C_{t}^{B}}{\Delta t}\right)^{2}+C_{c}^{2} \mathbf{u}^{h} \cdot \mathbf{G}_{c} \mathbf{u}^{h}+C_{c}^{2} \frac{\mathbf{B}^{h}}{\sqrt{\rho \mu_{0}}} \cdot \mathbf{G}_{c} \frac{\mathbf{B}^{h}}{\sqrt{\rho \mu_{0}}}+C_{\eta}^{2} \frac{\eta^{2}}{\mu_{0}^{2}}\left\|\mathbf{G}_{c}\right\|^{2}\right]^{-\frac{1}{2}}$ \\
\hline $\begin{array}{l}\text { Solenoidal } \\
\text { Constraint }\end{array}$ & $\hat{\tau}_{\psi}=1 /\left(C_{t}^{I} \operatorname{tr}\left(\mathbf{G}_{\mathbf{c}}\right) \hat{\tau}_{B}\right)$ \\
\hline
\end{tabular}

Definition of stabilization parameters used in stabilized equations, which use the contravarient metric tensor $\mathbf{G}_{c}$ (Equation (21)) to define an element-level streamwise length scale. In this study the values of the constants have been taken as $C_{t}^{V}=C_{c}=C_{\mu}=C_{B}=C_{t}^{B}=C_{\eta}=1.0$.

\subsection{Brief Overview of Discrete Systems of Equations}

To give context to the discussion of solution methods and linear algebra techniques that we employ in the linear solution methods above, we present a brief discussion of the structure of the equations that result from the FE discretization of the weak form of the resistive MHD equations. In the brief discussion below the focus is on the VMS terms generated in the both the induction equation and in the enforcement of the solenoidal constraint though the Lagrange multiplier, $\psi$. First the weak form of the solenoidal constraint is considered. The resulting stabilized form for this equation, in expanded form, is given by

$$
\begin{gathered}
F_{\psi}=\int_{\Omega} s(\nabla \cdot \mathbf{B}) d \Omega+ \\
\sum_{e} \int_{\Omega_{e}} \hat{\tau}_{I} \nabla s \cdot\left(\frac{\partial \mathbf{B}}{\partial t}+\nabla \cdot\left[\mathbf{u} \otimes \mathbf{B}-\mathbf{B} \otimes \mathbf{u}-\frac{\eta}{\mu_{0}}\left(\nabla \mathbf{B}-(\nabla \mathbf{B})^{T}\right)+\psi \mathbf{I}\right]\right) d \Omega .
\end{gathered}
$$

This expansion includes the weak form of a Laplacian operator acting on the Lagrange multiplier as,

$$
L_{\psi}=\sum_{e} \int_{\Omega_{e}} \hat{\tau}_{I} \nabla s \cdot \nabla \psi d \Omega
$$

which is produced by the stabilized formulation of the solenoidal constraint equation. It should be noted that this term is the analogue of the weak pressure Laplacian term $L_{P}=\sum_{e} \int_{\Omega_{e}} \rho \hat{\tau}_{m} \nabla \Phi \cdot \nabla P d \Omega$ generated in the total mass continuity equation (see the general discussion for stabilized FE CFD in [99] and our previous development in [25] in the context of 2D MHD). These VMS operators are critical in the elimination of oscillatory modes from the null space of the resistive MHD saddle point system for both $(\mathbf{u}, P)$ and $(\mathbf{B}, \psi)$ and allow equal-order interpolation of all the unknowns (see [99] for incompressible CFD and [41,42] for resistive MHD and a coercivity proof of stability).

The weak form of the magnetic induction equation, in expanded form, using the definition of $R_{\psi}$ for the solenoidal constraint is

$$
F_{I}=\int_{\Omega} \mathbf{C} \cdot \mathbf{R}_{I} d \Omega+\sum_{e} \int_{\Omega_{e}} \hat{\tau}_{I}\left(\mathbf{R}_{I} \otimes \mathbf{u}-\mathbf{u} \otimes \mathbf{R}_{I}\right): \nabla \mathbf{C} d \Omega+\sum_{e} \int_{\Omega_{e}} \hat{\tau}_{\psi}(\nabla \cdot \mathbf{C})(\nabla \cdot \mathbf{B}) d \Omega .
$$

In this form the existence of the weak divergence-type Laplacian operator is clearly evident. This term adds to the stability of the VMS form $[41,42]$ and also enhances the ability to iteratively invert the 
magnetic induction sub-block in the Jacobain matrix for our physics-based and approximate block factorization methods described in [100].

A finite element (FE) discretization of the stabilized equations gives rise to a system of coupled, nonlinear, non-symmetric algebraic equations, the numerical solution of which can be very challenging. These equations are linearized using an inexact form of Newton's method as described in Section 4.1. A formal block matrix representation of these discrete linearized equations is given by

$$
\left[\begin{array}{cccc}
\mathbf{F}_{\mathbf{u}} & \mathbf{B}^{\mathbf{T}} & \mathbf{Z} & \mathbf{0} \\
\mathbf{D} & \mathbf{L}_{\mathbf{P}} & \mathbf{0} & \mathbf{0} \\
\mathbf{Y} & \mathbf{0} & \mathbf{F}_{I} & \mathbf{B}^{\mathbf{T}} \\
\mathbf{0} & \mathbf{0} & \mathbf{B} & \mathbf{L}_{\psi}
\end{array}\right]\left[\begin{array}{c}
\delta \hat{\mathbf{u}} \\
\delta \hat{\mathbf{P}} \\
\delta \hat{\mathbf{B}} \\
\delta \hat{\psi}
\end{array}\right]=-\left[\begin{array}{c}
\mathbf{F}_{\mathbf{u}} \\
\mathbf{F}_{\mathbf{P}} \\
\mathbf{F}_{\mathbf{B}} \\
\mathbf{F}_{\psi}
\end{array}\right]
$$

where the block diagonal contribution of the stabilization procedure has been highlighted by a specific ordering. The block matrix, $\mathbf{F}_{\mathbf{u}}$, corresponds to the discrete transient, convection, diffusion and stress terms acting on the unknowns $\delta \hat{\mathbf{u}}$; the matrix, $\mathbf{B}$, corresponds to the discrete gradient operator; $\mathbf{D}$, the discrete representation of the continuity equation terms with velocity (note for a true incompressible flow this would be the divergence operator); the block matrix, $\mathbf{F}_{I}$, corresponds to the discrete transient, convection, diffusion acting on magnetic induction, and the matrices, $\mathbf{L}_{\mathbf{P}}, \mathbf{L}_{\psi}$, correspond to the discrete "pressure Laplacian" and "Lagrange multiplier Laplacian" discussed above. The right hand side vectors contain the residuals for Newton's method. The existence of the nonzero matrices, $\mathbf{L}_{\mathbf{P}}, \mathbf{L}_{\psi}$, in the stabilized FE discretization is in contrast to Galerkin methods using mixed interpolation that produce a zero block on the total mass continuity and solenoidal constraint diagonal. The existence of these block matrices helps to enable the solution of the linear systems with a number of algebraic and domain decomposition type preconditioners that rely on non-pivoting ILU type factorization, or in some cases methods such as Jacobi or Guass-Seidel as sub-domain solvers $[101,80]$.

The difficulty of producing robust and efficient preconditioners for Galerkin (as well as centered finite difference and non-staggered finite volume) formulations has motivated the use of many different types of decoupled solution methods. Often, transient schemes combine semi-implicit methods with fractional-step (operator splitting) approaches or use fully-decoupled solution strategies. In these cases, the motivation is to reduce memory usage and to produce a simplified equation set for which efficient solution strategies already exist. Unfortunately, these simplifications place significant limitations on the broad applicability of these methods. A detailed presentation of the characteristics of current solution methods is far beyond the scope of this brief overview. The intent of our method of fully-coupling the resistive MHD PDEs in the nonlinear solver is to preserve the inherently strong coupling of the physics with the goal to produce a more robust solution methodology. Preservation of this strong coupling, however, places a significant burden on the linear solution procedure to solve the fully coupled algebraic systems. Finally it should be noted that in our actual linear algebra implementation we use a specific ordering of the unknowns locally at each FE node with each degree of freedom ordered consecutively. A single coupled matrix problem, $\mathbf{J}_{\mathbf{s}}=-\mathbf{F}$, is solved at each Newton step with sophisticated algebraic domain decomposition and multilevel preconditioned Krylov methods as described in Section 4.2.

\section{References}

[1] H. Goedbloed, S. Poedts, Principles of Magnetohydrodynamics with Applications to Laboratory and Astrophysical Plasmas, Cambridge Univ. Press, 2004.

[2] W. Dai, P. R. Woodward, A simple finite difference scheme for multidimensional magnetohydrodynamic equations, J. Comput. Phys. 142 (1998) 331.

[3] D. Ryu, F. Miniati, T. W. Jones, A. Frank, A divergence-free upwind code for multi-dimensional magnetohydrodynamics flows, Astrophys. J. 509 (1998) 244. 
[4] D. S. Balsara, Divergence-free adaptive mesh refinement for magnetohydrodynamics, J. Comput. Phys. 174 (2001) 614-648.

[5] G. Tóth, The $\nabla \cdot \mathbf{B}=\mathbf{0}$ constraint in shock-capturing magnetohydrodynamics codes, J. Comput. Phys. $161(2000) 605-652$.

[6] G. Tóth, R. Keppens, M. A. Botchev, Implicit and semi-implicit schemes in the Versatile Advection Code: numerical tests, Astron. Astrophys. 332 (1998) 1159-1170.

[7] R. Keppens, G. Tóth, M. A. Botchev, A. V. D. Ploeg, Implicit and semi-implicit schemes: algorithms, Int. J. Numer. Meth. Fluids 30 (1999) 335-352.

[8] A. Y. Aydemir, D. C. Barnes, An implicit algorithm for compressible three-dimensional magnetohydrodynamic calculations., J. Comput. Phys. 59 (1) (1985) 108 - 19.

[9] W. Park, J. Breslau, J. Chen, G. Y. Fu, S. C. Jardin, S. Klasky, J. Menard, A. Pletzer, B. C. Stratton, D. Stutman, H. R. Strauss, L. E. Sugiyama, Nonlinear simulation studies of tokamaks and STS., Nuclear fusion 43 (6) (2003) $483-9$.

[10] S. C. Jardin, J. A. Breslau, Implicit solution of the four-field extended-magnetohydrodynamic equations using high-order high-continuity finite elements., Phys. Plasmas 12 (5) (2005) 056101.

[11] D. S. Harned, W. Kerner, Semi-implicit method for three-dimensional compressible magnetohydrodynamic simulation, J. Comput. Phys. 60 (1985) 62-75.

[12] D. D. Schnack, D. C. Barnes, D. S. Harned, E. J. Caramana, Semi-implicit magnetohydrodynamic calculations, J. Comput. Phys. 70 (1987) 330-354.

[13] D. S. Harned, Z. Mikic, Accurate semi-implicit treatment of the Hall effect in magnetohydrodynamic computations, J. Comput. Phys. 83 (1989) 1-15.

[14] C. R. Sovinec, A. H. Glasser, T. A. Gianakon, D. C. Barnes, R. A. Nebel, S. E. Kruger, D. D. Schnack, S. J. Plimpton, A. Tarditi, M. S. Chu, the NIMROD team, Nonlinear magnetohydrodynamics simulation using high-order finite elements, J. Comput. Phys. 195 (1) (2004) 355-386.

[15] A. Hujeirat, IRMHD: an implicit radiative and magnetohydrodynamical solver for self-gravitating systems, Mon. Not. R. Astron. Soc. 298 (1998) 310-320.

[16] A. C. Robinson, et. al., ALEGRA: An arbitrary Lagrangian-Eulerian multimaterial, multiphysics code, in: AIAA 2008-1235 46th AIAA Aerospace Sciences Meeting and Exhibit, Reno, NV, 2008.

[17] G. Toth, R. Keppens, Versatile advection code, http://www.phys.uu.nl/ toth/.

[18] M. M. Marinak, G. D. Kerbel, N. A. Gentile, O. Jones, S. P. D. Munro, T. R. Dittrich, S. W. Haan, Three-dimensional hydra simulations of national ignition facility targets, Phy. of Plasmas 8 (2001) 22752280 .

[19] L. Chacón, D. A. Knoll, J. M. Finn, Implicit, nonlinear reduced resistive MHD nonlinear solver, J. Comput. Phys. 178 (1) (2002) 15-36.

[20] L. Chacón, D. A. Knoll, J. M. Finn, An implicit nonlinear reduced resistive MHD solver, J. Comput. Phys. 178 (2002) 15-36.

[21] U. Shumlak, J. Loverich, Approximate riemann solver for the two-fluid plasma model, J. Comp. Phy. 187 (2003) 620-638.

[22] L. Chacón, A non-staggered, conservative, $\nabla \cdot \mathbf{B}=\mathbf{0}$, finite-volume scheme for 3D implicit extended magnetohydrodynamics in curvilinear geometries, Comput. Phys. Comm. 163 (2004) 143-171. 
[23] D. A. Knoll, L. Chacón, Coalescence of magnetic islands in the low-resistivity, Hall-MHD regime., Phys. Rev. Lett. 96 (13) (2006) $135001-4$.

[24] L. Chacón, An optimal, parallel, fully implicit newton-krylov solver for three-dimensional visco-resistive magnetohydrodynamics, Phys. Plasmas 15 (2008) 056103.

[25] J. N. Shadid, R. P. Pawlowski, J. W. Banks, L. Chacón, P. T. Lin, R. S. Tuminaro, Towards a scalable fully-implicit fully-coupled resistive MHD formulation with stabilized FE methods, J. Comput. Phys. 229 (20) (2010) 7649-7671.

[26] U. Shumlak, R. Lilly, N. Reddell, E. Sousa, B. Srinivasan, Advanced physics calculations using a multifluid plasma model, Computer Physics Comm. 182 (2011) 1767-1770.

[27] S. C. Jardin, Review of implicit methods for the magnetohydrodynamic description of magnetically confined plasmas, J. Comp. Physics 231822.

[28] J. Nedelec, Mixed finite elements in $\mathbf{R}^{3}$, Numerische Mathematik 35 (1980) 315-341.

[29] C. Evans, J. Hawley, Simulation of magnetohydrodynamic flows: a constrained transport method, The Astrophysical Journal 332 (1988) 659-677.

[30] J. Hyman, M. Shashkov, Adjoint operators for the natural discretizations of the divergence, gradient and curl on logically rectangular grids, Appl. Num. Math. 25 (1997) 413-442.

[31] P. B. Bochev, A. C. Robinson, Matching algorithms with physics: exact sequences of finite element spaces, in: D. Estep, S. Tavener (Eds.), Preservation of stability under discretization, Colorado State University, SIAM, Philadelphia, 2001, pp. 145-165.

[32] P. B. Bochev, J. J. Hu, A. C. Robinson, R. S. Tuminaro, Towards robust 3D Z-pinch simulations: discretization and fast solvers for magnetic diffusion in heterogeneous conductors, Electronic Transactions on Numerical Analysis 15, http://etna.msc.kent.edu. Special issue for the Tenth Copper Mountain Conference on Multigrid Methods.

[33] D. Schotzau, Mixed finite element methods for stationary incompressible magneto-hydrodynamics, Numerische Mathematik 96 (2004) 771800.

[34] L. Chacón, D. A. Knoll, J. M. Finn, Hall MHD effects in the 2-d Kelvin-Helmholtz / tearing instab lity, Phys. Lett. A 308 (2003) 187-197.

[35] S. Lankalapalli, J. Flaherty, M. Shephard, H. Strauss, An adaptive finite element method for magnetohydrodynamics, J. Comp. Phys. 225 (2007) 363-381.

[36] M. Gunzburger, A. Meir, J. Peterson, On the existence, uniqueness, and finite element approximation of solutions of the equations of stationary, incompressible magnetohydrodynamics, Mathematics of Computation 56 (3) (1991) 523563.

[37] J.-F. Gerbeau, A stabilized finite element method for the incompressible magnetohydrodynamic equations, Numer. Math. 87 (2000) 83-111.

[38] M. Costabel, M. Dauge, Singularities of electromagnetic fields in polyhedral domains, Archives for Rational Mechanics and Analysis 151 (3) (2000) 221276.

[39] M. Costabel, M. Dauge, Weighted regularization of maxwell equations in polyhedral domains, Numerische Mathematik 93 (2) (2002) 239277.

[40] N. B. Salah, A. Soulaimani, W. G. Habashi, M. Fortin, A conservative stabilized finite element method for the magento-hydrodyanamics equations, Int. J. Num. Meth. Fuilds 29 (1999) 535-554. 
[41] R. Codina, N. Hernández-Silva, Stabilized finite element approximation of the stationary magentohydrodyanamics equations, Comput. Mech. 38 (2006) 344-355.

[42] R. Codina, N. Hernández-Silva, Approximation of the thermally coupled mhd problem using a stabilized finite element method, J. Comp. Physics. 230 (2011) 1281-1303.

[43] F. Brezzi, On existence, uniqueness and approximation of saddle-point problems arising from Lagrange multipliers, RAIRO Model. Math. Anal. Numer. 21 (1974) 129-151.

[44] M. Gunzburger, Finite Element Methods for Viscous Incompressible Flows, Academic Press, Boston, 1989.

[45] S. Badia, R. Codina, R. Planas, On an unconditionally convergent stabilized finite element approximation of resistive magnetohydrodynamics, JCP 234 (2013) 399-416.

[46] A. Dedner, F. Kemm, D. Kroner, C.-D. Munz, T. Schnitzer, M. Wesenberg, Hyperbolic divergence cleaning for the mhd equations, J. Comp Physics 175 (2002) 645-673.

[47] R. Moreau, Magneto-hydrodynamics, Kluwer, Dordrecht, 1990.

[48] P. A. Davidson, An Introduction to Magnetohydrodynamics, Cambridge Univ. Press, 2001.

[49] H. R. Strauss, Nonlinear, 3-dimensional magnetohydrodynamics of noncircular tokamaks, Phys. Fluids 19 (1) (1976) 134-140.

[50] R. D. Hazeltine, M. Kotschenreuther, P. J. Morrison, A four-field model for tokamak plasma dynamics, Phys. Fluids 28 (8) (1985) 2466-2477.

[51] J. F. Drake, T. M. Antonsen, Nonlinear reduced fluid equations for toroidal plasmas, Phys. Fluids 27 (4) (1984) 898-908.

[52] A. N. Brooks, T. Hughes, Streamline upwind/petrov-galerkin formulations for convection dominated flows with particular emphasis on the incompressible navier-stokes equations, Comp. Meth. Appl. Mech. and Eng. 32 (1982) 199-259.

[53] T. Hughes, L. Franca, M. Balestra, A new finite element formulation for computational fluid dynamics: V. Circumventing the Babuska-Brezzi condition: A stable Petrov-Galerkin formulation of the Stokes problem accommodating equal-order interpolations, Comput. Meth. Appl. Mech. Engrg. 59 (1986) 85-99.

[54] T. Hughes, Multiscale phenomena: Green's functions, the dirichlet-to-neumann formulation, subgrid scale models, bubbles and the origins of stabilized methods, Comp. Meth. Appl. Mech. and Eng. 127 (1995) $387-401$.

[55] T. Hughes, G. Feijoo, L. Mazzei, J. Quincy, The variational multiscale method: A paradigm for computational mechanics, Comp. Meth. Appl. Mech. Engrg. 166 (1998) 3-24.

[56] T. J. R. Hughes, G. Scovazzi, 1. P. Franca, Chapter 2: Multiscale and Stabilized Methods in Encyclopedia of Computational Mechanics, Edited by Erwin Stein, Rene de Borst and Thomas J.R. Hughes. Volume 3: Fluids, John Wiley, 2007.

[57] E. C. Cyr, J. N. Shadid, R. S. Tuminaro, R. P. Pawlowski, L. Chacón, A new approximate block factorization preconditioner for 2D incompressible (reduced) resistive MHD, SISC 35 (2013) B701-B730.

[58] R. Codina, On stabilized finite element methods for linear systems of convection-diffusion-reaction equations, Comp. Meth. Appl. Mech. and Eng. 188 (2000) 61-88.

[59] R. Codina, Stabilized finite element approximation of transient incompressible flows using orthogonal subscales, Comp. Meth. App. Mech. Eng. 191 (2002) 4295-4321. 
[60] S. Collis, M. Heinkenschloss, Analysis of the streamline-upwind/petrov-galerkin method applied to the solution of optimal control problems, technical Report TR02-01, Department of Computational and Applied Mathematics, Rice University, Houston, TX 77005-1892 (2002).

[61] D. Sondak, J. N. Shadid, A. A. Oberai, R. P. Pawlowski, E. C. Cyr, T. M. Smith, A new class of finite element variational multiscale turbulence models for incompressible magnetohydrodynamics, J. Comp. Phys. 295 (2015) 596-616.

[62] T. Hughes, M. Mallet, A new finite element formulation for computational fluid dynamics: III. The generalized streamline operator for multidimentional advective-diffusive systems, Comput. Meth. Appl. Mech. Engrg. 58 (1986) 305-328.

[63] F. Shakib, Finite element analysis of the compressible Euler and Navier-Stokes equations, Ph.D. thesis, Division of Applied Mathematics, Stanford University (1989).

[64] J. U. Brackbill, D. C. Barnes, The effect of nonzero $\nabla \cdot \mathbf{B}$ on the numerical solution of the magnetohydrodynamic equations, J. Comput. Phys. 35 (3) (1980) 426-430.

[65] B. Marder, A method for incorporating gauss law into electromagnetic pic codes, J. Comp. Phy. 68 (1987) $48-55$.

[66] U. M. Ascher, L. R. Petzold, Computer Methods for Ordinary Differential Equations and DifferentialAlgebraic Equations, SIAM, 1998.

[67] J. E. Dennis, Jr., R. B. Schnabel, Numerical Methods for Unconstrained Optimization and Nonlinear Equations, Series in Automatic Computation, Prentice-Hall, Englewood Cliffs, NJ, 1983.

[68] P. N. Brown, Y. Saad, Convergence theory of nonlinear Newton-Krylov algorithms, SIAM J. Optimization 4 (1994) 297-330.

[69] R. S. Dembo, S. C. Eisenstat, T. Steihaug, Inexact Newton methods, SIAM J. Numer. Anal. 19 (1982) 400-408.

[70] S. C. Eisenstat, H. F. Walker, Choosing the forcing terms in an inexact Newton method, SIAM J. Sci. Comput. 17 (1996) 16-32.

[71] S. C. Eisenstat, H. F. Walker, Globally convergent inexact Newton methods, SIAM J. Optimization 4 (1994) 393-422.

[72] J. N. Shadid, R. S. Tuminaro, H. F. Walker, An inexact Newton method for fully-coupled solution of the Navier-Stokes equations with heat and mass transport, J. Comput. Phys. 137 (1997) 155-185.

[73] R. P. Pawlowski, J. N. Shadid, J. P. Simonis, H. F. Walker, Globalaization techniques for Newton-Krylov methods and applications to the fully-coupled solution of the Navier-Stokes equations, SIAM Review 48 (2006) 700-721.

[74] E. T. Phipps, R. P. Pawlowski, Efficient Expression Templates for Operator Overloading-based Automatic Differentiation, Vol. 87 of Lecture Notes in Computational Science and Engineering, Springer, 2012, pp. 309-320.

[75] M. Heroux, R. Bartlett, V. Howle, R. Hoekstra, J. Hu, T. Kolda, R. Lehoucq, K. Long, R. Pawlowski, E. Phipps, A. Salinger, H. Thornquist, R. Tuminaro, J. Willenbring, A. Williams, An Overview of Trilinos Project, ACM Trans. Math. Software 31 (3) (2005) 397-423.

[76] J. N. Shadid, A. G. Salinger, R. P. Pawlowski, P. T. Lin, G. L. Hennigan, R. S. Tuminaro, R. B. Lehoucq, Stabilized FE computational analysis of nonlinear steady state transport/reaction systems, Comp. Meth. Applied Mech. Eng. 195 (2006) 1846-1871. 
[77] Y. Saad, Iterative Methods for Sparse Linear Systems, SIAM, 2003.

[78] A. Quarteroni, A. Valli, Domain Decomposition Methods for Partial Differential Equations, Oxford University Press, Oxford, 1999.

[79] R. Tuminaro, C. Tong, J. Shadid, K.D.Devine, D. Day, On a multilevel preconditioning module for unstructured mesh Krylov solvers: two-level Schwarz, Comm. Num. Method. Eng. 18 (2002) 383-389.

[80] J. Shadid, R. Tuminaro, K. Devine, G. Henningan, P. Lin, Performance of fully-coupled domain decomposition preconditioners for finite element transport/reaction simulations, J. Comput. Phys. 205 (1) (2005) 24-47.

[81] U. Trottenberg, C. Oosterlee, A. Schüller, Multigrid, Academic Press, London, 2001.

[82] W. Hackbusch, Multigrid Methods and Applications, Vol. 4 of Computational Mathematics, SpringerVerlag, Berlin, 1985.

[83] U. Trottenberg, C. Oosterlee, A. Schüller, Multigrid, Academic Press, London, 2001.

[84] W. L. Briggs, V. E. Henson, S. McCormick, A Multigrid Tutorial, Second Edition, SIAM, Philadelphia, 2000 .

[85] T. Davis, Direct methods for sparse linear systems, SIAM, Philadelphia, PA, 2006.

[86] M. Sala, Amesos 2.0 reference guide, Tech. Rep. SAND2004-4820, Sandia National Laboratories (September 2004).

[87] J. Ruge, K. Stüben, Algebraic multigrid (AMG), in: S. F. McCormick (Ed.), Multigrid Methods, Vol. 3 of Frontiers in Applied Mathematics, SIAM, Philadelphia, PA, 1987, pp. 73-130.

[88] M. Gee, C. Siefert, J. Hu, R. Tuminaro, M. Sala, ML 5.0 smoothed aggregation user's guide, Tech. Rep. SAND2006-2649, Sandia National Laboratories, Albuquerque, NM, 87185 (2006).

[89] P. T. Lin, M. Sala, J. N. Shadid, R. S. Tuminaro, Performance of a geometric and an algebraic multilevel preconditioner for incompressible flow and transport, in: Computational Mechanics: WCCM VI in conjunction with APCOM’04, Tsinghus Univ. Press, Springer-Verlag, 2004.

[90] P. T. Lin, M. Sala, J. N. Shadid, R. S. Tuminaro, Performance of fully-coupled algebraic multilevel domain decomposition preconditioners for incompressible flow and transport, Int. J. Num. Meth. Eng. 67 (9) (2006) 208-225.

[91] P. T. Lin, J. N. Shadid, R. S. Tuminaro, M. Sala, G. L. Hennigan, R. P. Pawlowski, A parallel fullycoupled algebraic multilevel preconditioner applied to multiphysics PDE applications: Drift-diffusion, flow/transport/reaction, resistive MHD, Int. J. Num. Meth. Fluids 64 (2010) 1148-1179.

[92] P. T. Lin, J. N. Shadid, R. S. Tuminaro, M. Sala, Performance of a petrov-galerkin algebraic multilevel preconditioner for finite element modeling of the semiconductor device drift-diffusion equations, Int. J. Num. Meth. Eng. 84 (2010) 448-469.

[93] R. S. Tuminaro, M. Heroux, S. A. Hutchinson, J. N. Shadid, Aztec user's guide-version 2.1, Tech. Rep. Sand99-8801J, Sandia National Laboratories, Albuquerque NM, 87185 (Nov. 1999).

[94] G. K. Batchelor, An Introduction to Fluid Mechanics, Cambridge Univ. Press, 1967.

[95] V. M. Fadeev, I. F. Kvartskhava, N. N. Komarov, Self-focusing of local plasma currents., Nuclear fusion 5 (3) (1965) $202-209$.

[96] D. Biskamp, Magnetic Reconnection in Plasmas, Cambridge University Press, Cambridge, UK, 2000. 
[97] D. A. Knoll, L. Chacón, Coalescence of magnetic islands, sloshing, and the pressure problem., Phys. Plasmas 13 (3) (2006) 32307 - 1.

[98] L. Chacón, D. A. Knoll, J. M. Finn, Hall MHD effects in the 2-D Kelvin-Helmholtz/tearing instability, Phys. Lett.: A 308 (2-3) (2003) 187-197.

[99] J. Donea, A. Huerta, Finite Element Methods for Flow Problems, John Wiley, 2002.

[100] E. G. Phillips, E. C. Cyr, J. N. Shadid, R. P. Pawlowski, Approximate block preconditioners and effective Schur-complement approximations for the dual saddle-point problem of incompressible resistive MHD, in preperation.

[101] J. Shadid, A fully-coupled Newton-Krylov solution method for parallel unstructured finite element fluid flow, heat and mass transfer simulations, Int. J. CFD 12 (1999) 199-211. 